\title{
Stratified semiconcave control-Lyapunov functions and the stabilization problem
}

\author{
Ludovic Rifford \\ Institut Girard Desargues, Université Claude Bernard Lyon I, 43, Bd du 11 Novembre 1918, 69622 Villeurbanne cedex, France
}

Received 5 November 2003; received in revised form 26 March 2004; accepted 6 July 2004

Available online 21 March 2005

\begin{abstract}
Given a globally asymptotically controllable control system, we construct a control-Lyapunov function which is stratified semiconcave; that is, roughly speaking whose singular set has a Whitney stratification. Then we deduce the existence of smooth feedbacks which make the closed-loop system almost globally asymptotically stable.
\end{abstract}

(C) 2005 L'Association Publications de l'Institut Henri Poincaré. Published by Elsevier B.V. All rights reserved

Keywords: Control-Lyapunov function; Semiconcave function; Feedback stabilization

MSC: 93D05; 93D20; 93B05; 34D20; 49J52; 49L25; 70K15

\section{Introduction}

This paper is concerned with the stabilization problem for control systems of the form

$$
\dot{x}=f(x, \alpha):=\sum_{i=1}^{m} \alpha_{i} f_{i}(x),
$$

where $f_{1}, \ldots, f_{m}$ are smooth vector fields on $\mathbb{R}^{N}$ and where the control $\alpha=\left(\alpha_{1}, \ldots, \alpha_{m}\right)$ belongs to $\overline{B_{m}}$ the closed unit ball in $\mathbb{R}^{m}$. We focus on control systems which are globally asymptotically controllable.

Definition 1. The system (1) is said to be globally asymptotically controllable (abreviated GAC) if the two following conditions are satisfied:

E-mail address: rifford@igd.univ-lyon1.fr (L. Rifford). 
1. (Attractivity) For each $x \in \mathbb{R}^{N}$ there exists a control $\alpha(\cdot): \mathbb{R}_{\geqslant 0} \rightarrow \overline{B_{m}}$ such that the corresponding trajectory $x(\cdot ; \alpha, x)$ tends to 0.

2. (Lyapunov stability) For each $\epsilon>0$, there is a $\delta>0$ such that for each $x \in \mathbb{R}^{N}$ with $\|x\| \leqslant \delta$ there exists a control $\alpha(\cdot): \mathbb{R}_{\geqslant 0} \rightarrow \overline{B_{m}}$ such that the corresponding trajectory $x(\cdot ; \alpha, x)$ converges to the origin and satisfies $\|x(t ; \alpha, x)\| \leqslant \epsilon$ for all $t \geqslant 0$.

Given a GAC control system of the form (1), the purpose of the stabilization problem is to study the possible existence of a feedback $\alpha(\cdot): \mathbb{R}^{N} \mapsto \overline{B_{m}}$ which makes the closed-loop system

$$
\dot{x}=f(x, \alpha(x))=\sum_{i=1}^{m} \alpha_{i}(x) f_{i}(x),
$$

globally asymptotically stable. In the last twenty years this subject has been the focus of considerable research. ${ }^{1}$ It is well-known that continuous stabilizing feedbacks do not exist in general; there are globally asymptotically controllable control systems which admit no continuous stabilizing feedbacks. The first example of such a system was given in 1979 by Sussmann in [33]. Then, in 1983 Brockett [7] produced a topological necessary condition which makes obstruction to the existence of such regular feedbacks; this condition provided a number of counterexamples such as the famous nonholonomic integrator. Moreover in the case of affine control systems, Artstein related the existence of continuous stabilizing feedbacks to the existence of a smooth control-Lyapunov function. This latter result showed that a GAC affine control system which does not admit a continuous stabilizing feedback cannot have a smooth control-Lyapunov function. Therefore all these results plead for the design of discontinuous stabilizing feedbacks and also for a new concept of nonsmooth control-Lyapunov function. Many authors such as, Sussmann [33], Clarke, Ledyaev, Sontag and Subbottin [10], Ancona and Bressan [3], or Rifford [21,22,24] proved the existence of discontinuous stabilizing feedback laws under general assumptions. Among them, only $[10,21,22,24]$ made use of a nonsmooth control-Lyapunov function. In the present paper, our aim is to develop further the work which was initiated in these papers and to establish a strong link between the presence of a nonsmooth control-Lyapunov function and the construction of discontinuous stabilizing feedbacks. Moreover we also present a new kind of smooth stabilizing feedback which is of interest in the stabilization problem.

Definition 2. ${ }^{2}$ A control-Lyapunov function (abreviated CLF) for the system (1) is a continuous function $V: \mathbb{R}^{N} \rightarrow$ $\mathbb{R}$ which is positive definite, proper and such that it is a viscosity supersolution of the following Hamilton-Jacobi equation:

$$
\max _{\alpha \in \overline{B_{m}}}\{-\langle f(x, \alpha), D V(x)\rangle\}-V(x) \geqslant 0
$$

In 1983, Sontag [30] introduced the framework of nonsmooth CLF and proved the equivalence between global asymptotic controllability and the existence of a continuous control-Lyapunov function. Later, revealing the importance of semiconcavity in the design of discontinuous stabilizing feedbacks (in the spirit of [10]), we extended Sontag's Theorem and proved that every GAC control system admits a continuous CLF which is semiconcave outside the origin (see [23]). We utilized the semiconcavity property in order to construct feedbacks which make the closed-loop system globally asymptotically stable in the sense of Carathéodory. Our construction provided a simple way to design stabilizing feedback laws which were continuous on an open dense subset of the state space,

\footnotetext{
1 We recommend to the reader the historical accounts of Coron [13] and Sontag [31].

2 This definition takes into account the exponential decrease condition that we introduced in the framework of nonsmooth control-Lyapunov functions. Moreover we recall that the property (3) is equivalent to the following in terms of proximal subgradients

$$
\forall x \in \Omega, \forall \zeta \in \partial_{P} V(x), \quad \min _{\alpha \in \overline{B_{m}}}\{\langle f(x, \alpha), \zeta\rangle\} \leqslant-V(x)
$$
}


but it does not fully reveal the dynamics of the closed-loop system around the singularities. In [25], we showed that the exact comprehension of the singular set of a semiconcave CLF allows us to construct discontinuous stabilizing feedbacks for which we can classify the singular points. Unfortunately, we were only able to produce such a result in dimension two; our construction was related to the natural stratification of the singular set $\Sigma(V) \subset \mathbb{R}^{2}$ into a disjoint union of submanifolds of dimension 0 and 1 . Such a stratification of $\Sigma(V)$ is not imaginable in dimension greater than two. As a matter of fact, the singular set of a semiconcave function coinciding locally with the singular set of a concave function, we may let the reader imagine concave functions with very bad singular sets. This thought induces us to introduce a better type of semiconcavity. Our idea is to further regularize a semiconcave control-Lyapunov function into what we call a stratified semiconcave CLF which is, roughly speaking, a semiconcave function whose the singular set is a Whitney stratification (as introduced in [16]). We prove the following:

Theorem 3. If the system (1) is GAC then there exists a control-Lyapunov function which is stratified semiconcave on $\mathbb{R}^{N} \backslash\{0\}$.

As we shall see in Section 2.1, the knowledge of this new kind of control-Lyapunov functions facilitates the construction of discontinuous stabilizing feedbacks. In dimension two, it allows us to detail the nature of the singularities and then to better understand the behavior of the closed-loop system. As a consequence, it enables us to construct a new kind of smooth stabilizing feedback.

Definition 4. The closed-loop system (2) is said to be almost globally asymptotically stable at the origin (abreviated AGAS) if the two following properties are satisfied:

(i) (Attractivity) For almost every $x \in \mathbb{R}^{N}$, the solution of (2) starting at $x$ converges to the origin.

(ii) (Lyapunov stability) For each $\epsilon>0$, there exists $\delta>0$ such that for each $x \in \mathbb{R}^{N}$ with $\|x\| \leqslant \delta$ the solution of (2) starting at $x$ satisfies $\|x(t)\| \leqslant \epsilon$ for all $t \geqslant 0$.

This kind of asymptotic stability was recently studied by Rantzer who produced a converse Lyapunov-like theorem. Indeed he related the almost asymptotic stability to the existence of a density function which plays the role of a Lyapunov function for the closed-loop system. In addition, he proved in a first paper $[19,20]$ some interesting consequences of the existence of such a density function. In the present paper, our approach is to consider a stratified semiconcave control-Lyapunov function in order to construct an almost globally asymptotically stabilizing feedback. Our CLF does not correspond in any case to Rantzer's density function but it allows us to contruct explicitly an AGAS feedback. Our second main result is the following:

Theorem 5. Assume that $N=2$. If the system (1) is GAC then there exists a feedback $\alpha(\cdot): \mathbb{R}^{N} \rightarrow \mathbb{R}^{m}$ (with $\alpha(0)=0)$ which is continuous on $\mathbb{R}^{N}$, of class $C^{1}$ outside the origin and such that the closed-loop system (2) is AGAS.

In fact, this results holds in any dimension. The construction of the feedback that we present in this paper is clearly detailed in the case of dimension two. Of course, this construction can be adapted in greater dimension but it would be much more intricate. Furthermore, at the end of the paper, we show that Theorem 5 can be extended in the framework of manifolds, we discuss what happens in the case of control systems with drift, and finally, we compare almost global asymptotic stability with other kinds of asymptotic stability.

Notations: $\quad$ Throughout this paper, $\mathbb{R}$ denotes the set of real numbers, $\|\cdot\|$ the Euclidean norm of $\mathbb{R}^{N}, B_{N}$ the open ball $\{x:\|x\|<1\}$ in $\mathbb{R}^{N}, \overline{B_{N}}$ the closure of $B_{N}$ and $B_{N}(x, r)=x+r B_{N}$ (resp. $\overline{B_{N}(x, r)}=x+r \overline{B_{N}}$ ) the ball 
(resp. the closed ball) centered at $x$ and with radius $r$. In addition $\mathbb{S}^{N-1}$ denotes the $(N-1)$-dimensional sphere in $\mathbb{R}^{N}$ and $S_{N}(x, r)$ denotes the sphere centered at $x$ with radius $r$ in $\mathbb{R}^{N}$. If $A$ is a subset of $\mathbb{R}^{N}$ then $\operatorname{int}(A)$ denotes the interior of $A$ and $\operatorname{co} A$ its convex hull. For any positive integer $n, \mathcal{L}^{n}$ denotes Lebesgue measure of dimension $n$ and if $\mathcal{K}$ is a finite set then $|\mathcal{K}|$ denotes the cardinality of the set $\mathcal{K}$. Furthermore, an admissible control for the system (1) is a Lebesgue-integrable function $\alpha(\cdot):[0, T] \rightarrow \overline{B_{m}}$ on some interval $[0, T]$. If $\alpha(\cdot):[0, T] \rightarrow \overline{B_{m}}$ is an admissible control, a trajectory for $\alpha(\cdot)$ is an absolutely continuous curve $x(\cdot):[0, T] \rightarrow \mathbb{R}^{N}$ such that

$$
\dot{x}(t)=\sum_{i=1}^{m} \alpha_{i}(x(t)) f_{i}(x(t))
$$

for almost all $t \in[0, T]$. If $x_{0}$ is some given state in $\mathbb{R}^{N}$ and if $\alpha(\cdot)$ is an admissible control, we denote by $x\left(\cdot ; x_{0}, \alpha\right)$ the trajectory solution of the system above and such that $x\left(0 ; x_{0}, \alpha\right)=x_{0}$. We denote by $\mathcal{A}$ the set of open-loop from $[0, \infty)$ controls into the control set $\overline{B_{m}}$. Moreover since we will take a "subdifferential" (or "superdifferential") point of view in this paper, we refer the reader to the book of Clarke et al. [11] for the definitions of proximal suband superdifferentials $\left(\partial_{P} V\right.$ and $\left.\partial^{P} V\right)$, of limiting subgradients $\partial_{L} V$ and of generalized gradients $\partial V$ of some function $V: \mathbb{R}^{N} \rightarrow \mathbb{R}$.

\section{Further regularization of a semiconcave CLF}

Throughout this section $\Omega$ is an open subset of $\mathbb{R}^{N}$.

\subsection{Preliminary results on semiconcave functions}

In this section we recall the definition and the basic properties of semiconcave functions which follow mainly from the decomposition of such functions into the sum of a concave function and a smooth ${ }^{3}$ function. We refer the reader to the forthcoming book of Cannarsa and Sinestrari [8] for a detailed study of semiconcavity.

Definition 6. A function $u: \Omega \rightarrow \mathbb{R}$ is said to be semiconcave on $\Omega$ if it is continuous on $\Omega$ and if for any $x_{0} \in \Omega$ there are constants $\rho, C>0$ such that

$$
\frac{1}{2}(u(x)+u(y))-u\left(\frac{x+y}{2}\right) \leqslant C\|x-y\|^{2},
$$

for all $x, y \in x_{0}+\rho B_{N}$.

Note that if the constant $C$ vanishes in (4), we recognize the usual definition of concave functions. As we shall see in the sequel, the properties of semiconcave functions are intimately related to those of concave functions. In Definition 6 since the constants $\rho$ and $C$ depend upon the point $x_{0} \in \Omega$, some authors (e.g. Cannarsa, Sinestrari) refer sometimes to local semiconcavity. We prefer to adopt Definition 6 and to speak about global $C$-semiconcavity whenever the constant $C$ of (4) does not depend upon $x_{0}$. In any case, semiconcavity implies Lipschitz continuity (see [8] for a proof of this result).

Proposition 7. Every semiconcave function $u: \Omega \rightarrow \mathbb{R}$ is locally Lipschitz on $\Omega$.

The local Lipschitz property can also be seen as a consequence of the following fundamental property of semiconcave functions. Any semiconcave function can be seen locally as the sum of a concave and a smooth function. Refering to global semiconcavity, we can state the result as follows:

\footnotetext{
3 In this paper, "smooth" means "of class $C^{\infty}$ ".
} 
Proposition 8. If $u$ is globally $C$-semiconcave on $\Omega$ and if in addition $\Omega$ is convex, then the function

$$
x \mapsto u(x)-4 C\|x\|^{2} \quad \text { is concave. }
$$

Hence the function $u$ can be written as the sum of a concave function and a smooth (quadratic) function:

$$
u(x)=\left[u(x)-4 C\|x\|^{2}\right]+4 C\|x\|^{2} .
$$

Proof. The function $x \mapsto g(x):=u(x)-4 C\|x\|^{2}$ is clearly continuous. Moreover for any $x, y \in \Omega$, we have

$$
\begin{aligned}
\frac{1}{2}(g(x)+g(y))-g\left(\frac{x+y}{2}\right) & =\frac{1}{2}(u(x)+u(y))-u\left(\frac{x+y}{2}\right)-2 C\left[\|x\|^{2}+\|y\|^{2}\right]+C\|x+y\|^{2} \\
& \leqslant C\|x-y\|^{2}+C\|x+y\|^{2}-2 C\left[\|x\|^{2}+\|y\|^{2}\right] \quad \text { by (4) } \\
& \leqslant 0,
\end{aligned}
$$

by the parallelogram identity; the result follows.

As in the case of Lipschitz functions, a function which is semiconcave on a compact set is in fact globally semiconcave on this set. This property is a (not so easy) consequence of the definition of semiconcavity. It permits us to extend the Proposition 8 to the case of nonconvex open sets.

Proposition 9. If $u$ is semiconcave on $\Omega$ then for any compact convex set $K \subset \Omega$, there exists a global constant $C_{K} \geqslant 0$ such that $u$ is globally $C_{K}$-semiconcave on $K$. Hence the function $u$ can be written on $K$ as the sum of a concave and a quadratic function:

$$
\forall x \in K, \quad u(x)=\left[u(x)-4 C_{K}\|x\|^{2}\right]+4 C_{K}\|x\|^{2} .
$$

This result does hold only on compact subsets of $\Omega$ which are convex. (Convex or concave properties of functions do have no meaning on sets which are not convex.) However we will see later (see Theorem 12) that the decomposition as a sum of a concave function and a smooth function remains valid on convex open sets. We shall now present some results concerning the superdifferentials of semiconcave functions. By classical properties of the superdifferentials of concave functions, the decomposition of semiconcave functions that we gave in Proposition 8 implies that the generalized gradient of a semiconcave function equals its proximal and viscosity superdifferentials (we refer the reader to the now classical references [11,6] for the definition of both these superdifferentials). Let us state the result (see [8] for its proof):

Proposition 10. If $u: \Omega \rightarrow \mathbb{R}$ is semiconcave then for any $x \in \Omega, \partial u(x)=\partial^{P} u(x)$, i.e. for any $\zeta \in \partial u(x)$,

$$
u(y)-u(x)-4 C\|y-x\|^{2} \leqslant\langle\zeta, y-x\rangle, \quad \forall y \in x+\rho B_{N},
$$

where $\rho$ and $C$ are the constants given in Definition 6.

On the other hand, still from the decomposition of Proposition 8, since the generalized gradient of a convex function is a monotone operator, so is the operator $x \mapsto-\partial u(x)+8 C x$. We can state the result as follows:

Proposition 11. If $u: \Omega \rightarrow \mathbb{R}$ is semiconcave then for any $x, y \in \Omega$, for any $\zeta_{x} \in \partial u(x)$, for any $\zeta_{y} \in \partial u(y)$,

$$
\left\langle-\zeta_{y}+\zeta_{x}, y-x\right\rangle \geqslant-8 C\|y-x\|^{2} .
$$




\subsection{Semiconcave functions on convex sets}

The purpose of this section is to present some initial results on the regularization of semiconcave functions. We prove that every semiconcave function on an open convex set can be approximated by the sum of a piecewise affine concave function and a smooth function. The technique of proof that we develop here will be very helpful when we shall regularize semiconcave functions on nonconvex domains. Before giving our result of approximation, we need the following fundamental theorem:

Theorem 12. If the open set $\Omega$ is convex then for any semiconcave function $u$ on $\Omega$ there exists two functions $g, \Psi: \Omega \rightarrow \mathbb{R}$ such that

$$
u=g+\Psi,
$$

where $g$ is concave and $\Psi$ is smooth.

Proof. Let us first assume that $\Omega=\mathbb{R}^{N}$. For every $n \geqslant 2$, the function $u$ is semiconcave on the ball $n \overline{B_{N}}:=$ $\overline{B_{N}(0, n)}$, hence there exists a constant $C_{n-1}$ such that the function $u(\cdot)-C_{n-1}\|x\|^{2}$ is concave on $n \overline{B_{N}}$ (by Proposition 9). Without loss of generality the sequence $\left(C_{n}\right)_{n \in \mathbb{N}}$ can be supposed to be strictly increasing. Set for any $x \in \Omega$,

$$
h_{1}(x):=C_{1}\|x\|^{2},
$$

and

$$
h_{n}(x):=C_{n}\|x\|^{2}+\sum_{i=1}^{n-1} i^{2}\left[C_{i}-C_{i+1}\right] \quad \text { if } n \geqslant 2 .
$$

By construction, we note that for each $n \geqslant 1$, the function $h_{n}$ is convex and that for any $n \geqslant 1$, the function $u-h_{n}$ is concave on $(n+1) \overline{B_{N}}$. In addition we have that for any $n \geqslant 1$,

$$
h_{n+1}(x) \leqslant h_{n}(x) \Longleftrightarrow x \in n \bar{B}_{N} .
$$

We define the function $h: \mathbb{R}^{N} \rightarrow \mathbb{R}$ by

$$
h(x):=\max _{n \in \mathbb{N}}\left\{h_{n}(x)\right\} \quad \text { for } x \in \mathbb{R}^{N} .
$$

By (8) the function $h$ is well defined (on $\Omega$ ) and moreover it is convex as a maximum of convex functions. On the other hand, we can note that for any $x \in \Omega$,

$$
u(x)-h(x)=u(x)-\max _{n \in \mathbb{N}}\left\{h_{n}(x)\right\}=u(x)+\min _{n \in \mathbb{N}}\left\{-h_{n}(x)\right\}=\min _{n \in \mathbb{N}}\left\{u(x)-h_{n}(x)\right\} .
$$

By (8) we deduce that on every $n \geqslant 1$, the function $u-h$ coincides with the function $\min \left\{u-h_{1}, \ldots, u-h_{n}\right\}$ on $n \overline{B_{N}}$. Let $x \in \Omega$, denote by $n_{0}$ the minimum over all the integers $n \geqslant 1$ such that $x \in n \overline{B_{N}}$. By (8) we get that for any $y \in B_{N}(x, 1)$,

$$
u(y)-h(y)=\min \left\{u(y)-h_{n_{0}-1}(y), u(y)-h_{n_{0}}(y), u(y)-h_{n_{0}+1}(y)\right\} .
$$

The three functions which appear in the minimum above are concave on $B_{N}(x, 1)$ hence $u-h$ is concave on this ball. Consequently $u-h$ is concave on all the balls $B_{N}(x, 1)$ for $x \in \Omega$, hence it is concave on $\Omega$. Unfortunately, the constructed function $h$ is not smooth. But if we consider a function $f: \mathbb{R}_{\geqslant 0} \rightarrow \mathbb{R}$, then we leave it to the reader to verify that, whenever the function $u$ is globally $C$-semiconcave on $\Omega$, the function $u-f\left(\|\cdot\|^{2}\right)$ is concave if and only if for any $x, y \in \Omega$,

$$
f\left(\left\|\frac{x+y}{2}\right\|^{2}\right)-\frac{1}{2}\left(f\left(\|x\|^{2}\right)+f\left(\|y\|^{2}\right)\right) \leqslant-C\|y-x\|^{2},
$$


that is if and only if the function $x \mapsto f\left(\|x\|^{2}\right)-4 C\|x\|^{2}$ is convex. We conclude that if $f$ is a smooth function on $\Omega$ such that for any $n \geqslant 1$, the function $f\left(\|\cdot\|^{2}\right)-4 C_{n}\|\cdot\|^{2}$ is convex on $n B_{N}$ then the function $u-f\left(\|\cdot\|^{2}\right)$ is globally concave on $\Omega$. The construction of the function $f$ is left to the reader. Now return to the general case of an open set $\Omega$ in $\mathbb{R}^{N}$. By classical results on convex sets we know that any open convex set of $\mathbb{R}^{N}$ is diffeomorphic to $\mathbb{R}^{N}$, hence there exists a smooth diffeomorphism $\phi: \mathbb{R}^{N} \rightarrow \Omega$. Moerover this diffeomorphism can be taken to preserve convexity; if $g: R^{N} \rightarrow \Omega$ is a convex function then the function $g \circ \phi^{-1}: \Omega \rightarrow \mathbb{R}$ is convex too. So we look at the function $u \circ \phi$ on $\mathbb{R}^{N}$ which is semiconcave as the composition of a smooth function and a semiconcave function. By the result above this function can be written as the sum of a concave and a smooth function: $u \circ \phi=g+\Psi$. Thus the function $g \circ \phi^{-1}$ is concave, the function $\Psi \circ \phi^{-1}$ is smooth, and in addition

$$
u=g \circ \phi^{-1}+\Psi \circ \phi^{-1}
$$

which gives the result.

Corollary 13. If the open set $\Omega$ is convex and if $u: \Omega \rightarrow \mathbb{R}$ is semiconcave then for any continuous function $\epsilon: \Omega \rightarrow(0, \infty)$ such that for every $x \in \Omega, B_{N}(x, \epsilon(x)) \subset \Omega$, there exists a piecewise affine concave function $g_{\epsilon}: \Omega \rightarrow \mathbb{R}$ and a smooth function $\Psi_{\epsilon}: \Omega \rightarrow \mathbb{R}$ such that for every $x \in \Omega$,

(i) $\left|u(x)-g_{\epsilon}(x)-\Psi_{\epsilon}(x)\right| \leqslant \epsilon(x)$,

(ii) $\partial_{P} g_{\epsilon}(x)+\nabla \Psi_{\epsilon}(x) \subset \partial_{P} u\left(x+\epsilon(x) B_{N}\right)+\epsilon(x) B_{N}$.

Proof. Without loss of generality we can assume that $\epsilon(\cdot)<1$ and that for every $x \in \Omega, \overline{B_{N}(x, \epsilon(x))} \subset \Omega$. Moreover by continuity of the function $\epsilon$ there exists some new continuous function $\tilde{\epsilon}: \Omega \rightarrow(0,1)$ such that

$$
\forall x \in \Omega, \forall y \in B_{N}(x, \epsilon(x)), \quad \tilde{\epsilon}(x) \leqslant \epsilon(y) .
$$

By the previous theorem, there exist two functions $g, \Psi: \Omega \rightarrow \mathbb{R}$ with $g$ concave and $\Psi$ smooth such that $u=g+\Psi$ on $\Omega$. Define two functions $\Psi_{\epsilon}, \tilde{g}: \Omega \rightarrow \mathbb{R}$ by $\Psi_{\epsilon}(x):=\Psi(x)+\|x\|^{2}$ and $\tilde{g}(x):=g(x)-\|x\|^{2}$. Of course $\Psi_{\epsilon}$ is smooth, $\tilde{g}$ is concave and we have,

$$
u=\tilde{g}+\Psi_{\epsilon} .
$$

By concavity, the function $\tilde{g}$ is locally Lipschitz on $\Omega$, hence for every $x \in \Omega$, there exists $L_{\tilde{g}, x} \geqslant 1$ such that $\tilde{g}$ is $L_{\tilde{g}, x}$-Lipschitz on $\overline{B_{N}(x, \epsilon(x))} \subset \Omega$. Moreover since the function $\Psi_{\epsilon}$ is smooth, there exists as well for each $x \in \Omega$ some constant $L_{\Psi_{\epsilon}, x} \geqslant 1$ such that $\nabla \Psi_{\epsilon}(\cdot)$ is $L_{\Psi_{\epsilon}, x}$-Lipschitz on $\overline{B_{N}(x, \epsilon(x))} \subset \Omega$. Denote for each $x \in \Omega$ by $L_{x}$ the maximum of both constants $L_{\tilde{g}, x}, L_{\Psi_{\epsilon}, x}$. (Notice that the function $x \mapsto L_{x}$ can be constructed to be locally finite in $\Omega$.)

By a classical density theorem (see for instance [11, Theorem 3.1, p. 39]), the proximal subdifferentials of $\tilde{g}$ are nonempty on a dense subset of $\Omega$. Therefore if we denote ${ }^{4}$ by $D$ the set of $x \in \Omega$ such that $\partial_{P} \tilde{g}(x) \neq \emptyset$, we have

$$
\Omega \subset \bigcup_{x \in D} B_{N}\left(x, \frac{\tilde{\epsilon}(x)^{2}}{8 L_{x}^{3}}\right) .
$$

(This inclusion holds because of the local finiteness of the function $x \mapsto L_{x}$ in $\Omega$.)

The local compactness of $\Omega$ implies that there exists a locally finite family $\left(x_{n}\right)_{n \in \mathbb{N}}$ in $D$ such that

$$
\Omega \subset \bigcup_{n \in \mathbb{N}} B_{N}\left(x_{n}, \frac{\tilde{\epsilon}\left(x_{n}\right)^{2}}{8 L_{x_{n}}^{3}}\right) .
$$

\footnotetext{
4 Notice that since the function $\tilde{g}$ is concave, at each point $x$ such that $\partial_{P} \tilde{g}(x) \neq \emptyset$ it is differentiable (the converse being false).
} 
Fix $n \in \mathbb{N}$ and define the function $h_{n}: \Omega \rightarrow \mathbb{R}$ by

$$
h_{n}(x):=\tilde{g}\left(x_{n}\right)+\left\langle\nabla \tilde{g}\left(x_{n}\right), x-x_{n}\right\rangle \text {. }
$$

Lemma 14. We have the two following inequalities:

(i) $\forall x \in \Omega, h_{n}(x) \geqslant \tilde{g}(x)$,

(ii) $\forall x \in B_{N}\left(x_{n}, \tilde{\epsilon}\left(x_{n}\right)^{2} /\left(8 L_{x_{n}}^{3}\right)\right), h_{n}(x) \leqslant \tilde{g}(x)+\tilde{\epsilon}\left(x_{n}\right)^{2} /\left(4 L_{x_{n}}^{2}\right)$.

Proof. Since $x_{n} \in D$, we have that $\left\{\nabla \tilde{g}\left(x_{n}\right)\right\}=\partial^{P} \tilde{g}\left(x_{n}\right)$. Thus Proposition 10 implies the first inequality. Let us prove the second inequality. For any $x \in B_{N}\left(x_{n}, \tilde{\epsilon}\left(x_{n}\right)^{2} /\left(8 L_{x_{n}}^{3}\right)\right)$, we have

$$
h_{n}(x)-\tilde{g}(x)=\tilde{g}\left(x_{n}\right)-\tilde{g}(x)+\left\langle\nabla \tilde{g}\left(x_{n}\right), x-x_{n}\right\rangle=\left\langle\zeta, x_{n}-x\right\rangle+\left\langle\nabla \tilde{g}\left(x_{n}\right), x-x_{n}\right\rangle,
$$

with $\zeta \in \operatorname{co}\left(\partial \tilde{g}\left(\left[x, x_{n}\right]\right)\right)$ (by Lebourg's Theorem, see for instance [9, Theorem 2.3.7, p. 41] or [11, Theorem 2.4, p. 75]). We conclude easily by Cauchy-Schwarz inequality.

We set on $\Omega$ the following function:

$$
\forall x \in \Omega, \quad g_{\epsilon}(x):=\min _{n \in \mathbb{N}}\left\{h_{n}(x)\right\} .
$$

By Lemma 14(i)-(ii), the function $g_{\epsilon}$ is well-defined and then concave. Let us first prove assertion (i) in the statement of Corollary 13. that

By (11) for every $x \in \Omega$, there exists $n \in \mathbb{N}$ such that $x \in B_{N}\left(x_{n}, \tilde{\epsilon}\left(x_{n}\right)^{2} /\left(8 L_{x_{n}}^{3}\right)\right)$. Hence Lemma 14(ii) implies

$$
g_{\epsilon}(x) \leqslant \tilde{g}(x)+\frac{\tilde{\epsilon}\left(x_{n}\right)^{2}}{4 L_{x_{n}}^{2}} \leqslant \tilde{g}(x)+\epsilon(x) \quad \text { (by (9)). }
$$

In consequence, Lemma 14(i) combined with (10) gives (i). It remains to prove (ii). We need the following lemma:

Lemma 15. For every $x \in \Omega$, there exists $n_{0} \in \mathbb{N}$ such that $\left\|x-x_{n_{0}}\right\| \leqslant\left(\tilde{\epsilon}\left(x_{n_{0}}\right)\right) /\left(2 L_{x_{n_{0}}}\right)$ and $g_{\epsilon}(x)=h_{n_{0}}(x)$.

Proof. By concavity of $g$ on $\Omega$, for every $n \in \mathbb{N}$ we have

$$
\forall x \in \Omega, \quad g(x) \leqslant g\left(x_{n}\right)+\left\langle\nabla g\left(x_{n}\right), x-x_{n}\right\rangle .
$$

We deduce that for every $n \in \mathbb{N}$ and for any $x \in \Omega$,

$$
\begin{aligned}
\tilde{g}(x)-h_{n}(x) & =g(x)-\|x\|^{2}-\tilde{g}\left(x_{n}\right)-\left\langle\nabla \tilde{g}\left(x_{n}\right), x-x_{n}\right\rangle \\
& =g(x)-\|x\|^{2}-g\left(x_{n}\right)+\left\|x_{n}\right\|^{2}-\left\langle\nabla g\left(x_{n}\right)-2 x_{n}, x-x_{n}\right\rangle \\
& \leqslant-\|x\|^{2}+\left\|x_{n}\right\|^{2}+2\left\langle x_{n}, x-x_{n}\right\rangle=-\left\|x-x_{n}\right\|^{2} .
\end{aligned}
$$

Hence if $x \in \Omega$ satisfies $\left\|x-x_{n}\right\|^{2}=\delta^{2}>\tilde{\epsilon}\left(x_{n}\right)^{2} /\left(4 L_{x_{n}}^{2}\right)$ then $\tilde{g}(x) \leqslant h_{n}(x)-\delta^{2}$, which by (12) implies

$$
g_{\epsilon}(x) \leqslant h_{n}(x)-\delta^{2}+\frac{\tilde{\epsilon}\left(x_{n}\right)^{2}}{4 L_{x_{n}}^{2}}<h_{n}(x) .
$$

This proves that for each $x$ in $\Omega$ the minimum in the definition of $g_{\epsilon}(x)$ is not attained for $h_{j}(x)$ with $\| x-$ $x_{j} \|>\tilde{\epsilon}\left(x_{j}\right) /\left(2 L_{x_{j}}\right)$. Therefore since the family $\left(x_{n}\right)_{n \in \mathbb{N}}$ is locally finite and since for any $x \in \Omega$ the compact set $\overline{B_{N}(x, \epsilon(x))}$ is included in $\Omega$, this means that for any $x \in \Omega$ the function $g_{\epsilon}$ can be written as a minimum of a finite number of affine functions $h_{n}$ in $B_{N}(x, \epsilon(x))$. This proves the lemma. 
Fix $\bar{x} \in \Omega$ and $\bar{\zeta} \in \partial_{P} g_{\epsilon}(\bar{x})$. By elementary properties of proximal subdifferentials (recall that we refer to [11] for a complete presentation of proximal analysis) if the minimum in the definition of $g_{\epsilon}(\bar{x})$ is attained and if $\partial_{P} g_{\epsilon}(\bar{x}) \neq \emptyset$ then $g_{\epsilon}$ is differentiable at $\bar{x}$ and

$$
\partial_{P} g_{\epsilon}(\bar{x})=\left\{\nabla g_{\epsilon}(\bar{x})\right\}=\left\{\nabla h_{n_{0}}(\bar{x})\right\}=\left\{\nabla \tilde{g}\left(x_{n_{0}}\right)\right\}=\partial_{P} \tilde{g}\left(x_{n_{0}}\right),
$$

where $n_{0}$ is such that $g_{\epsilon}(\bar{x})=h_{n_{0}}(\bar{x})$. Hence since $\left\|x_{n_{0}}-\bar{x}\right\|<\tilde{\epsilon}(\bar{x}) /\left(2 L_{x_{n_{0}}}\right)$ we can write

$$
\begin{aligned}
\bar{\zeta}+\nabla \Psi_{\epsilon}(\bar{x}) & \in \partial_{P} \tilde{g}\left(x_{n_{0}}\right)+\nabla \Psi_{\epsilon}(\bar{x}) \\
& \subset \partial_{P} \tilde{g}\left(x_{n_{0}}\right)+\nabla \Psi_{\epsilon}\left(x_{n_{0}}\right)+\left\|\bar{x}-x_{n_{0}}\right\| L_{x_{n_{0}}} \overline{B_{N}} \\
& \subset \partial_{P} u\left(\bar{x}+\epsilon(\bar{x}) B_{N}\right)+\epsilon(\bar{x}) B_{N} \quad(\text { by }(9)) .
\end{aligned}
$$

The proof of Corollary 13 is complete.

Note that in the proof of Corollary 13, we have been able to control the distance $\left\|x-x_{n_{0}}\right\|$ in Lemma 15 because we worked with $\tilde{g}$ instead of $g$. Our argument was based on the fact that $\tilde{g}$ is the sum of a concave function $(g)$ and of a strictly concave function $\left(-\|\cdot\|^{2}\right)$. Since we have still the proof of Corollary 13 in mind, we present and prove a related result which will be very useful in the sequel. (In the statement of Proposition $16, B_{\infty}$ denotes the open ball of $\mathbb{R}^{N}$ relative to the infinity norm $\|\cdot\|_{\infty}$.)

Proposition 16. Let $\Omega$ be an open set in $\mathbb{R}^{N}$ and $u: \Omega \rightarrow \mathbb{R}$ be a semiconcave function. Let $K$ be a compact cube in $\Omega$ and $\epsilon$ be a constant in $(0,1)$ such that $\overline{K+\epsilon B_{\infty}} \subset \Omega$. If $C$ is some positive constant such that the function $g:=u-C\|\cdot\|^{2}$ is concave on $\overline{K+\epsilon B_{\infty}}$, then there exists $\tilde{g}_{\epsilon}: \Omega \rightarrow \mathbb{R}$ such that

(i) for every $x \in K, \tilde{g}_{\epsilon}(x)+(C+1)\|x\|^{2} \leqslant u(x)$,

(ii) for every $x \in \overline{K+\epsilon B_{\infty}}, u(x)-\epsilon \leqslant \tilde{g}_{\epsilon}(x)+(C+1)\|x\|^{2} \leqslant u(x)+\epsilon$,

(iii) for every $x \in \overline{K+\epsilon B_{\infty}}, \partial_{P} \tilde{g_{\epsilon}}(x)+2(C+1) x \subset \partial_{P} u\left(x+\epsilon B_{\infty}\right)+\epsilon B_{\infty}$,

(iv) the function $\tilde{g}_{\epsilon}$ is a piecewise affine concave function on $\mathbb{R}^{N}$,

(v) $\forall x \in \Omega, x \notin \overline{K+\epsilon B_{\infty}} \Longrightarrow \tilde{g}_{\epsilon}(x)+(C+1)\|x\|^{2}>u(x)$.

Proof. We leave it to the reader to show that there exists a smooth function $k: \mathbb{R}^{N} \rightarrow \mathbb{R}$ which satisfies the following properties:

(p1) for every $x \in K, k(x)=0$,

(p2) for every $x \notin \overline{K+\epsilon B_{\infty}}, k(x)>\frac{\epsilon^{2}}{4}$,

(p3) for every $x \in \mathbb{R}^{N}, k(x) \in\left[0, \frac{\epsilon}{2}\right]$

(p4) for every $x \in \overline{K+\epsilon B_{\infty}},\|\nabla k(x)\| \leqslant \frac{\epsilon}{2}$,

(p5) for every $x \in \mathbb{R}^{N}, \operatorname{Hess}_{x} k<I_{N}$,

where $\operatorname{Hess}_{x} k$ denotes the Hessian matrix of the function $k$ at $x$ and $I_{N}$ denotes the identity matrix in $M_{N}(\mathbb{R})$.

Define the function $\tilde{g}: \Omega \rightarrow \mathbb{R}$ by

$$
\forall x \in \Omega, \quad \tilde{g}(x):=g(x)-\|x\|^{2}+k(x)=\left(g(x)-\frac{1}{2}\|x\|^{2}+k(x)\right)-\frac{1}{2}\|x\|^{2} .
$$

Since the Hessian matrix of the function $\frac{1}{2}\|\cdot\|^{2}$ equals $I_{N}$, the property (p5) implies that the function $x \mapsto$ $\frac{1}{2}\|x\|^{2}-k(x)$ is convex on $\mathbb{R}^{N}$, and hence that the function $x \mapsto g(x)-\frac{1}{2}\|x\|^{2}+k(x)$ is concave on $\overline{K+\epsilon B_{\infty}}$. In consequence as shown by (14), the function $\tilde{g}$ can be written as the sum of a concave function and of a strictly con- 
cave function (the function $x \mapsto-\frac{1}{2}\|x\|^{2}$ ). Thus by the remark that we made just after the proof of Corollary 13, one can design a finite family $\left(x_{i}\right)_{i \in I}$ of points in $\overline{K+\epsilon B_{\infty}}$ such that if we set for every $i \in I$ the function

$$
h_{i}(x):=\tilde{g}\left(x_{i}\right)+\left\langle\nabla \tilde{g}\left(x_{i}\right), x-x_{i}\right\rangle
$$

and if we define the function $\hat{g}$ by $\hat{g}(x):=\min _{i \in I} h_{i}(x)$, then the following lemma holds:

Lemma 17. For every $x \in \overline{K+\epsilon B_{\infty}}$, the following properties are satisfied:

(i) $\tilde{g}(x) \leqslant \hat{g}(x) \leqslant \tilde{g}(x)+\frac{\epsilon^{2}}{4}$,

(ii) there exists $i_{0} \in I$ such that $\left\|x-x_{i_{0}}\right\| \leqslant \frac{\epsilon}{4(C+1)}$ and $\hat{g}(x)=h_{i_{0}}(x)$.

Set for every $x \in \Omega$

$$
\tilde{g}_{\epsilon}(x):=\hat{g}(x)-\frac{\epsilon^{2}}{4},
$$

and let us prove that the five assertions of Proposition 16 are satisfied.

The function $\tilde{g}_{\epsilon}(x)$ being a minimum of a finite number of affine functions, it is a piecewise affine concave function on $\mathbb{R}^{N}$. Which gives (iv). On ther other hand, assertions (i), (ii) and (v) are straightforward consequences of Lemma 17 and (p1)-(p3). It remains to prove assertion (iii).

Fix $\bar{x} \in \Omega$, and $\bar{\zeta} \in \partial_{P} \tilde{g}_{\epsilon}(\bar{x})$. As in the proof of Corollary 13, we know by construction of $\hat{g}$ and by Lemma 17(ii) that there exists $i_{0} \in I$ such that

$$
\left\|\bar{x}-x_{i_{0}}\right\| \leqslant \frac{\epsilon}{4(C+1)}
$$

and

$$
\partial_{P} \tilde{g}_{\epsilon}(\bar{x})=\left\{\nabla \tilde{g}\left(x_{i_{0}}\right)\right\}=\partial_{P} \tilde{g}\left(x_{i_{0}}\right) .
$$

Hence we can write

$$
\begin{aligned}
\bar{\zeta}+2(C+1) \bar{x} & \in \partial_{P} \tilde{g}\left(x_{i_{0}}\right)+2(C+1) \bar{x} \quad(\text { by }(16)) \\
& \subset \partial_{P} \tilde{g}\left(x_{i_{0}}\right)+2(C+1) x_{i_{0}}+2(C+1)\left\|\bar{x}-x_{i_{0}}\right\| \overline{B_{N}} \\
& \subset \partial_{P} g\left(x_{i_{0}}\right)+\nabla k\left(x_{i_{0}}\right)+2 C x_{i_{0}}+2(C+1)\left\|\bar{x}-x_{i_{0}}\right\| \overline{B_{N}} \\
& \subset \partial_{P} u\left(\bar{x}+\epsilon B_{N}\right)+\epsilon B_{N} \quad(\text { by }(15) \text { and }(\mathrm{p} 4)) \\
& \subset \partial_{P} u\left(\bar{x}+\epsilon B_{\infty}\right)+\epsilon B_{\infty},
\end{aligned}
$$

because $B_{N} \subset B_{\infty}$. Therefore the proof of Proposition 16 is complete.

\subsection{Singular set of semiconcave functions}

Let $\Omega$ be an open set of $\mathbb{R}^{N}$ and let $u: \Omega \rightarrow \mathbb{R}$ be a semiconcave function. By Rademacher's theorem we know that $u$ is differentiable almost everywhere in $\Omega$. Let us denote by $\Sigma(u)$ the singular set of $u$, i.e. the set of points of $\Omega$ where $u$ is not differentiable. We can also see $\Sigma(u)$ as the set of $x \in \Omega$ such that $\operatorname{dim}(\partial u(x)) \geqslant 1$; this point of view leads to a natural partition of the singular set. As a matter of fact, following moreorless the seminal work of Alberti, Ambrosio and Cannarsa [2], $\Sigma(u)$ can be written as the disjoint union of $N$ sets $\Sigma^{k}(u)$ (for $k \in\{1, \ldots, N\}$ ) defined by

$$
\Sigma^{k}(u):=\{x \in \Omega: \operatorname{dim}(\partial u(x))=k\} .
$$


Alberti et al. proved that for any $k \in\{1, \ldots, N\}$, the set $\Sigma^{k}(u)$ is countably $\mathcal{H}^{n-k}$-rectifiable, i.e. it is contained (up to a $\mathcal{H}^{n-k}$-negligible set) in a countable union of $C^{1}$ hypersurfaces of dimension $N-k$. But each set $\Sigma^{k}(u)$ is certainly not an exact hypersurface (or submanifold) of $\Omega$. As we noticed in [25], these sets cannot be realized to be smooth submanifolds of $\Omega$. We saw that even in dimension two, it is difficult to decompose the singular set $\Sigma(u)$ into a union of Lipschitz and $C^{1}$-submanifolds. And of course, this decomposition does not hold in greater dimension for general semiconcave functions. Our strategy is to approximate a given semiconcave function $u$ by a semiconcave function with a singular set regular enough. In addition if the initial function $u$ is a supersolution of some Hamilton-Jacobi equation then we would like that the constructed approximation remains almost a supersolution of the same Hamilton-Jacobi equation. After giving the definition of stratified semiconcave functions, we present our approximation results.

Let $Z$ be a closed subset of $\Omega$, and suppose that

$$
Z=\bigcup_{l \in\{0, \cdot, N\}} \bigcup_{i \in \mathcal{S}_{l}} S_{i}^{l}
$$

This decomposition is a Whitney stratification of $Z$ provided:

- The family $\left\{S_{i}^{l}\right\}_{l, i}$ is a locally finite collection of disjoint locally closed subsets called pieces or strata.

- Each stratum $S_{i}^{l}$ is a locally closed ${ }^{5}$ connected smooth submanifold of $\Omega$ of dimension $l$.

- $S_{i}^{l} \cap \overline{S_{j}^{k}} \neq \emptyset \Rightarrow l<k$ and $S_{i}^{l} \subset \overline{S_{j}^{k}}$.

- Whenever $S_{i}^{l}$ and $S_{j}^{k}$ are two strata with $l<k$ then the pair satisfies Whitney's conditions A and B: Suppose $\left(x_{n}\right)_{n} \in S_{j}^{k}$ is a sequence of points converging to some $y \in S_{i}^{l}$, suppose $\left(y_{n}\right)_{n} \in S_{i}^{l}$ also converge to $y$, and suppose that the secant lines $L_{n}=\left(x_{n} y_{n}\right)$ converge to some limiting line $l$, and the tangent spaces $T_{x_{n}} S_{j}^{k}$ converge to some limiting vector subspace $\tau$. Then
(A) $T_{y} S_{i}^{l} \subset \tau$.
(B) $l \subset \tau$.

We can now present the concept of stratified semiconcave function.

Definition 18. Let $u: \Omega \rightarrow \mathbb{R}$ be a semiconcave function; $u$ will be said to be stratified semiconcave (on $\Omega$ ) if the following conditions are satisfied:

(i) the set $\Sigma(u)$ is a Whitney stratification such that the strata of dimension $N-k$ are the connected components of $\Sigma^{k}(u)$

(ii) for every stratum $S$ of $\Sigma(u)$, the set $\bar{S}$ is a smooth manifold with boundary;

(iii) for every stratum $S$ of $\Sigma(u)$, the function $u$ is smooth on $\bar{S}$;

(iv) for every $x \in \Sigma^{k}(u)$, the set $\partial u(x)$ is a convex compact set of dimension $k$ with exactly $k+1$ extreme points $\zeta_{1}(x), \ldots, \zeta_{k+1}(x) .{ }^{6}$ In addition, for any stratum $S$ of $\Sigma^{k}(u)$ the maps $\zeta_{1}(\cdot), \ldots, \zeta_{k+1}(\cdot)$ are smooth on $S$ and moreover they can be smoothly extended to a neighborhood of $\bar{S}$ in such a way that $\operatorname{co}\left\{\zeta_{1}(x), \ldots, \zeta_{k+1}(x)\right\}$ remains a convex compact set of dimension $k$ with exactly $k+1$ extreme points for any $x \in \bar{S}$.

Let us make one remark of importance concerning the shape of the singular set of a stratified semiconcave function. Begin by the two-dimensional case.

\footnotetext{
5 We recall that a subset $X$ of $\mathbb{R}^{N}$ is said to be locally closed if it is of the form $X=C \cap O$ where $C$ is closed and $O$ is open.

6 In particular, this means that the limiting subgradient $\partial_{L} u(x)$ equals the set $\left\{\zeta_{1}(x), \ldots, \zeta_{k+1}(x)\right\}$.
} 


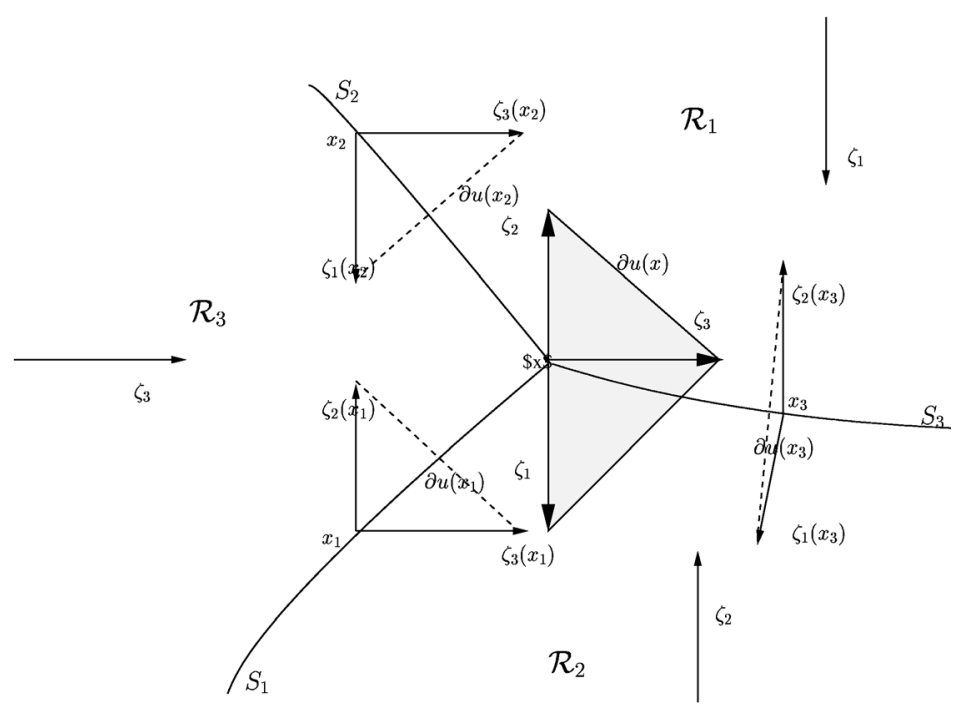

Fig. 1. Propagation of singularities in dimension two.

Let $u: \Omega \rightarrow \mathbb{R}$ be a stratified semiconcave function where $\Omega$ is an open subset of $\mathbb{R}^{2}$. We have

$$
\Sigma(u)=\Sigma^{1}(u) \cup \Sigma^{2}(u) .
$$

Since $u$ is stratified semiconcave on $\Omega$, the set $\Sigma^{1}(u)$ is a locally finite union of disjoint smooth submanifolds of dimension 1 and the set $\Sigma^{2}(u)$ is a discrete set of points in $\Omega$. Let us describe what happens in a small ball around some point $x \in \Sigma^{2}(u)$. There are three vectors $\zeta_{1}, \zeta_{2}, \zeta_{3} \in \mathbb{R}^{2}$ such that

$$
\partial u(x)=\operatorname{co}\left\{\zeta_{1}, \zeta_{2}, \zeta_{3}\right\}
$$

the full triangle with $\zeta_{1}, \zeta_{2}$ and $\zeta_{3}$ as extreme points. By properties of propagation of singularities (see [1, Lemma 4.5, p. 728]), each of the edges of this triangle (i.e., the segments $\left[\zeta_{2}, \zeta_{3}\right],\left[\zeta_{1}, \zeta_{3}\right],\left[\zeta_{1}, \zeta_{2}\right]$ ) is limit of the generalized gradients of points of a strata of dimension one. In other terms, this means that there exist three smooth submanifolds of dimension one $S_{1}, S_{2}$ and $S_{3}$ in $\Sigma^{1}(u)$ which satisfy the following properties:

For each $i=1,2,3$, the point $x$ belongs to the closure of $S_{i}$. Moreover if $\left(x_{n}\right)_{n}$ is some sequence of points in $S_{i}$ which converges to $x$, then the sequence of segments $\left(\partial u\left(x_{n}\right)\right)_{n}$ converges to the segments with endpoints $\left(\zeta_{j}\right)_{j}$ where $j \in\{1,2,3\} \backslash\{i\}$.

On Fig. 1, we show three points $x_{1}, x_{2}, x_{3}$ with generalized gradients $\partial u\left(x_{1}\right), \partial u\left(x_{2}\right), \partial u\left(x_{3}\right)$ which are very close to the edges of the triangle. On each stratum $S_{i}(i=1,2,3)$ the generalized gradient of $y \in S_{i}$ tends to an edge of the triangle $\partial u(x)$ as $y$ tends to $x$. This means that in a small ball centered at $x$, the complement of $\Sigma(u)$ can be divided into three regions $\mathcal{R}_{1}, \mathcal{R}_{2}$ and $\mathcal{R}_{3}$ where $u$ is smooth with a gradient close to $\zeta_{1}$ in $\mathcal{R}_{1}$, to $\zeta_{2}$ in $\mathcal{R}_{2}$ and to $\zeta_{3}$ in $\mathcal{R}_{3}$.

Consider now the general $N$-dimensional case. Let $u: \Omega \rightarrow \mathbb{R}$ be a stratified semiconcave function where $\Omega$ is an open subset of $\mathbb{R}^{N}$. In this case, we have

$$
\Sigma(u)=\Sigma^{1}(u) \cup \Sigma^{2}(u) \cup \cdots \cup \Sigma^{N}(u) .
$$


So, let $S$ be a stratum of $\Sigma(u)$ of dimension $N-k$ with $k \geqslant 2$ and let $x \in S$. The convex set $\partial u(x)$ is indeed a simplex of dimension $k \geqslant 2$ in $\mathbb{R}^{N}$. Hence if for every $l \in\{0, \ldots, k\}$ we denote by $F_{l}$ the number of faces ${ }^{7}$ of dimension $l$, we have:

$$
F_{l}=\left(\begin{array}{c}
k+1 \\
l+1
\end{array}\right)
$$

This means for example that $\partial u(x)$ has $k+1$ faces of dimension $k-1$ and $k+1$ faces of dimension zero, that is $k+1$ extreme points. Again by properties of propagation of singularities (see [1, Theorem 5.2, p. 732]), since the function $u$ is stratified semiconcave it turns out that for every $l \in\{1, \ldots, k-1\}$ there is a one-to-one correspondence between the set of faces of dimension $l$ and a certain subset of strata of $\Sigma^{l}(u)$. Let $l \in\{1, \ldots, k-1\}$, denote by $C_{1}^{l}, \ldots, C_{F_{l}}^{l}$ the $F_{l}$ faces of $\partial u(x)$ of dimension $l$; this means that there are exactly $F_{l}$ strata $S_{1}, \ldots, S_{F_{l}}$ in $\Sigma^{l}(u)$ such that for every $i=1, \ldots, F_{l}$ the following property is satisfied:

The point $x$ belongs to the closure of the stratum $S_{i}$. Moreover if $\left(x_{n}\right)_{n}$ is a sequence of points in $S_{i}$ which converges to $x$ then the sequence of convex sets $\left(\partial u\left(x_{n}\right)\right)_{n}$ converges to the face $C_{i}^{l}$.

The first example of stratified semiconcave function is the one of piecewise affine semiconcave functions. In this particular case, each set $\Sigma^{k}(u)$ is a disjoint union of open polyhedra of dimension $N-k$. Let us state the properties of such functions in the following proposition:

Proposition 19. Let be given $\left(h_{i}\right)_{i \in I}$ a finite family of affine functions in $\mathbb{R}^{N}$. If $u: \mathbb{R}^{N} \rightarrow \mathbb{R}$ is defined by

$$
u:=\min _{i \in I}\left\{h_{i}\right\}
$$

then it is a stratified semiconcave function and moreover it satisfies:

(i) For every $k \in\{1, \ldots, N\}$, the set $\Sigma^{k}(u)$ is a finite disjoint union of open polyhedra of dimension $N-k$.

(ii) For every $k \in\{1, \ldots, N\}$, the multivalued map $x \mapsto \partial u(x)=\partial^{P} u(x)$ is constant on each connected component of $\Sigma^{k}(u)$.

The proof of this result being straightforward, it is left to the reader.

If we consider a function defined as the minimum of a finite family of smooth functions, then it is semiconcave. However it is not necessarily stratified semiconcave. Indeed in the next section, we will see that this kind of functions is generically stratified semiconcave. This result comes from a transversality argument.

\subsection{Proof of Theorem 3}

In fact we will prove a more precise version of Theorem 3. Let us state the result that we prove.

Theorem 20. Let $V$ be a semiconcave control-Lyapunov function for the control system (1). Then for any continuous function $\epsilon: \mathbb{R}^{N} \backslash\{0\} \rightarrow(0, \infty)$, there exists a continuous function $V_{\epsilon}: \mathbb{R}^{N} \rightarrow \mathbb{R}$ which is stratified semiconcave on $\mathbb{R}^{N} \backslash\{0\}$, which is a viscosity supersolution of

$$
\max _{\alpha \in \overline{B_{m}}}\{-\langle f(x, \alpha), D V(x)\rangle\}-V(x)+\epsilon(x) \geqslant 0,
$$

and such that for any $x \in \mathbb{R}^{N} \backslash\{0\}$,

$$
\left|V_{\epsilon}(x)-V(x)\right| \leqslant \epsilon(x) .
$$

\footnotetext{
7 A face of a convex set $C$ is a convex subset $C^{\prime}$ such that every (closed) line segment in $C$ with an interior point in $C^{\prime}$ has both endpoints in $C^{\prime}$. We refer the reader to the textbook [29, Part IV, Section 18] for additional informations about faces of convex sets.
} 
Theorem 3 is a direct consequence of Theorem 20. As a matter of fact, if we apply Theorem 20 to a controlLyapunov function with $\epsilon=\frac{V}{2}$, then we obtain some stratified semiconcave function $V_{\epsilon}$ which satisfies the conclusions of Theorem 20 and hence which is positive definite and proper from (18). Hence if we set $\widetilde{V}_{\epsilon}:=V_{\epsilon}^{2}$ we get a veritable stratified semiconcave CLF as in Theorem 3. It remains to prove Theorem 20; this proof will occupy the next eight pages.

In order to simplify the proof, we will work with balls of the $\infty$-norm. Let us denote by $\|x\|_{\infty}$ the $\infty$-norm of $x \in \mathbb{R}^{N}$, and by $B_{\infty}$ (resp. $\overline{B_{\infty}}$ ) the open unit ball (resp. the closed ball) of $\mathbb{R}^{N}$ relative to this norm.

Set $\Omega:=\mathbb{R}^{N} \backslash\{0\}$. We can decompose $\Omega$ into a "quasi" partition of compact cubes. There exists a family $\left(x_{i}\right)_{i \in \mathbb{N}}$ of points in $\Omega$ and a family $\left(\rho_{i}\right)_{i \in \mathbb{N}}$ of positive real numbers such that the three following properties hold:

(P1) $\Omega=\bigcup_{i \in \mathbb{N}} \overline{B_{\infty}\left(x_{i}, \rho_{i}\right)}=\bigcup_{i \in \mathbb{N}} K_{i}\left(\right.$ where $\left.K_{i}:=\overline{B_{\infty}\left(x_{i}, \rho_{i}\right)}\right)$.

(P2) For each $i \in \mathbb{N}$, the compact set $\overline{K_{i}+\rho_{i} B_{\infty}}$ is included in $\Omega$, and the covering $\Omega=\bigcup_{i \in \mathbb{N}} \overline{K_{i}+\rho_{i} B_{\infty}}$ is locally finite.

(P3) If $K_{i} \cap K_{j} \neq \emptyset$ then $K_{i}$ and $K_{j}$ have a common face, i.e. there is a face $F_{i}$ of $K_{i}$ and a face $F_{j}$ of $K_{j}$ such that $\left(K_{i} \backslash F_{i}\right) \cap\left(K_{j} \backslash F_{j}\right)=\emptyset$ and either $F_{i} \subset F_{j}$ either $F_{j} \subset F_{i}$.

By (P2) for each $i \in I$, we denote by $L_{V}^{i} \geqslant 1$ a Lipschitz constant of $V$ on the compact cube $\overline{K_{i}+\rho_{i} B_{\infty}}$, by $M_{i}$ the maximum of $\|f(x, \alpha)\|$ for $x \in \overline{K_{i}+\rho_{i} B_{\infty}}$ and $\alpha \in \overline{B_{N}}$, by $L_{f}^{i}$ the Lipschitz constant of the multivalued map $x \mapsto f\left(x, \overline{B_{m}}\right)$ on $\overline{K_{i}+\rho_{i} B_{\infty}}$ and by $\epsilon_{i}$ the minimum of the function $\epsilon(\cdot)$ on $\overline{K_{i}+\rho_{i} B_{\infty}}$. For each $i \in I$, we set

$$
\mu_{i}:=\min \left\{\frac{\epsilon_{i}}{2\left(L_{V}^{i} L_{f}^{i}+L_{V}^{i}+M_{i}\right)}, \min \left\{\frac{\rho_{j}}{10}: j \text { s.t. } K_{i} \cap K_{j} \neq \emptyset\right\}\right\} .
$$

Notice that by construction, if $x \in \Omega$ belongs to some compact set $\overline{K_{i}+\mu_{i} B_{\infty}}$, then

$$
\mu_{i} \leqslant \frac{\epsilon(x)}{2}
$$

Moreover notice also that by (P2), we have that for every $i \in I$

$$
K_{i}+2 \mu_{i} B_{\infty} \subset \overline{K_{i}+\rho_{i} B_{\infty}} \subset \Omega .
$$

Thus by Proposition 8 , for every $i \in I$ there exists a positive constant $C_{i}$ such that

$$
V(x)=\left[V(x)-C_{i}\|x\|^{2}\right]+C_{i}\|x\|^{2}=g_{i}(x)+\Psi_{i}(x),
$$

where $g_{i}:=V-C_{i}\|\cdot\|^{2}$ is concave on $\overline{K_{i}+2 \mu_{i} B_{\infty}}$ and $\Psi_{i}:=C_{i}\|\cdot\|^{2}$ is smooth on the same set. By Proposition 16 , for each $i \in I$ there exists a piecewise affine concave function $G_{i}: \mathbb{R}^{N} \rightarrow \mathbb{R}$ such that the following properties hold:

(a) for every $x \in K_{i}, G_{i}(x)+\left(C_{i}+1\right)\|x\|^{2} \leqslant V(x)$,

(b) for every $x \in \overline{K_{i}+\mu_{i} B_{\infty}}, V(x)-\mu_{i} \leqslant G_{i}(x)+\left(C_{i}+1\right)\|x\|^{2} \leqslant V(x)+\mu_{i}$,

(c) for every $x \in \overline{K_{i}+\mu_{i} B_{\infty}}, \partial_{P} G_{i}(x)+2\left(C_{i}+1\right) x \subset \partial_{P} V\left(x+\mu_{i} B_{\infty}\right)+\mu_{i} B_{\infty}$,

(d) there exists a finite set $J^{i}$ and some vectors $\left(H_{j}^{i}\right)_{j \in J^{i}}$ in $K_{i}$ and some constants $\left(\hat{h}_{j}^{i}\right)_{j \in J^{i}}$ such that

$$
\forall x \in \mathbb{R}^{N}, \quad G_{i}(x)=\min _{j \in J^{i}}\left\{h_{i}^{j}(x)\right\},
$$

where $h_{i}^{j}(x)=\left\langle H_{i}^{j}, x\right\rangle+\hat{h}_{i}^{j}$.

(e) for every $x \notin \overline{K_{i}+\mu_{i} B_{\infty}}, G_{i}(x)>g_{i}(x)-\|x\|^{2}$. 
Define for every $i \in I$ the function $V_{i}: \mathbb{R}^{N} \rightarrow \mathbb{R}$ by

$$
V_{i}(x):=G_{i}(x)+\Psi_{i}(x)+\|x\|^{2}=G_{i}(x)+\left(C_{i}+1\right)\|x\|^{2} .
$$

The function $V_{i}$ is the sum of the concave function $G_{i}$ and of the smooth function $\Psi_{i}+\|\cdot\|^{2}$, hence it is semiconcave on $\mathbb{R}^{N}$. Fix $x$ in $\overline{K_{i}+\mu_{i} B_{\infty}}$ and $\zeta$ in $\partial_{P} V_{i}(x)$.

By (c) there exists $\bar{x} \in B_{\infty}\left(x, \mu_{i}\right)$ such that $\zeta \in \partial V(\bar{x})+\mu_{i} B_{\infty}$; this means that there exist $\bar{x} \in K_{i}+2 \mu_{i} B_{\infty}$, $\bar{\zeta} \in \partial V(\bar{x})$ and $\bar{v} \in B_{\infty}$ such that

$$
\zeta=\bar{\zeta}+\mu_{i} \bar{v}
$$

Since $V$ is a CLF for the system (1), it is a supersolution of the Hamilton-Jacobi equation (3) and hence

$$
\max _{\alpha \in \overline{B_{m}}}\{-\langle f(\bar{x}, \alpha), \bar{\zeta}\rangle\}-V(\bar{x}) \geqslant 0 .
$$

Therefore by construction of the constants $L_{f}^{i}, L_{V}^{i}, M_{i},(23)$ and (21) give

$$
\begin{aligned}
\max _{\alpha \in \overline{B_{m}}}\{-\langle f(x, \alpha), \zeta\rangle\} & \geqslant \max _{\alpha \in \overline{B_{m}}}\{-\langle f(x, \alpha), \bar{\zeta}\rangle\}-\mu_{i} M_{i} \\
& \geqslant \max _{\alpha \in \overline{B_{m}}}\{-\langle f(\bar{x}, \alpha), \bar{\zeta}\rangle\}-L_{f}^{i}\|x-\bar{x}\|\|\zeta\|-\mu_{i} M_{i} \\
& \geqslant V(\bar{x})-\left[L_{f}^{i} L_{V}^{i}+M_{i}\right] \mu_{i} \geqslant V(x)-\left[L_{f}^{i} L_{V}^{i}+L_{V}^{i}+M_{i}\right] \mu_{i} \\
& \geqslant V(x)-\frac{\epsilon(x)}{2} \quad \text { (by (19)). }
\end{aligned}
$$

Consequently we proved that for every $x \in \overline{K_{i}+\mu_{i} B_{\infty}}$ and for every $\zeta \in \partial_{P} V_{i}(x)$,

$$
\max _{\alpha \in \overline{B_{m}}}\{-\langle f(x, \alpha), \zeta\rangle\}-V(x)+\frac{\epsilon(x)}{2} \geqslant 0 .
$$

On the other hand by (a) and (e) we have that $V_{i}(x) \leqslant V(x)$ for every $x \in K_{i}$ and that $V_{i}(x)>V(x)$ whenever $x \notin \overline{K_{i}+\mu_{i} B_{\infty}}$. Set for every $x \in \mathbb{R}^{N}$,

$$
\widetilde{V}(x):=\inf _{i \in \mathbb{N}}\left\{V_{i}(x)\right\} .
$$

By (P1) and the remark above, for every $x \in \Omega$ we have

$$
\widetilde{V}(x)=\inf _{i \text { s.t. } x \in \overline{K_{i}+\mu_{i} B_{\infty}}}\left\{V_{i}(x)\right\} .
$$

Hence since the family $\left\{\overline{K_{i}+\mu_{i} B_{\infty}}\right\}_{i \in \mathbb{N}}$ is locally finite in $\Omega$, this means that for every $x \in \Omega$ there exists $i_{x} \in \mathbb{N}$ such that $x \in \overline{K_{i_{x}}+\mu_{i_{x}} B_{\infty}}$ and $\widetilde{V}(x)=V_{i_{x}}(x)$. In particular (b) implies

$$
V(x)-\mu_{i_{x}} \leqslant \widetilde{V}(x) \leqslant V(x)+\mu_{i_{x}} \Longrightarrow V(x)-\frac{\epsilon(x)}{2} \leqslant \tilde{V}(x) \leqslant V(x)+\frac{\epsilon(x)}{2} \quad \text { (by (20)). }
$$

The function $\widetilde{V}$ is semiconcave on $\Omega$ (as a locally finite infimum of semiconcave functions), and in addition (24) and (25) imply that for every $x \in \Omega$ and for every $\zeta \in \partial_{P} \widetilde{V}(x),{ }^{8}$

$$
\max _{\alpha \in \overline{B_{m}}}\{-\langle f(x, \alpha), \zeta\rangle\}-V(x) \geqslant-\frac{\epsilon(x)}{2} \Longrightarrow \max _{\alpha \in \overline{B_{m}}}\{-\langle f(x, \alpha), \zeta\rangle\}-\widetilde{V}(x) \geqslant-\epsilon(x) \quad \text { (by (25)). }
$$

\footnotetext{
8 Recall that if some function $f: \Omega \rightarrow \mathbb{R}$ is defined by $f(x):=\min _{i \in I}\left\{g_{i}(x)\right\}$ where $I$ is a finite set and $g_{1}, \ldots, g_{I}$ some continuous functions on $\Omega$. Then for every $x \in \Omega$ and for every $\zeta \in \partial_{P} f(x)$, there exists $i \in I$ such that $f(x)=g_{i}(x)$ and $\zeta \in \partial_{P} g_{i}(x)$.
} 
To summarize, the function $\widetilde{V}$ is a semiconcave control-Lyapunov function which satisfies the properties (18) and (17). Unfortunately, it is not necessarily stratified semiconcave. Properties (a) and (e) imply that the function $\widetilde{V}$ coincides with $V_{i}$ on each cube $\overline{B_{\infty}\left(x_{i}, \rho_{i}-\delta_{i}\right)}$, where $\delta_{i}$ denotes the maximum of the $\mu_{j}$ 's for $K_{i} \cap K_{j} \neq \emptyset$ (note that $\rho_{i}-\delta_{i} \geqslant \frac{9 \rho_{i}}{10}>0$ by (19)). In particular, we deduce that the function $\widetilde{V}$ is stratified semiconcave (by Proposition 19) on each open cube $B_{\infty}\left(x_{i}, \rho_{i}-\delta_{i}\right)$. In the sets $K_{i} \backslash B_{\infty}\left(x_{i}, \rho_{i}-\delta_{i}\right)$, the function $\widetilde{V}$ is a minimum of quadratic functions, so it is not necessarily stratified semiconcave. We shall prove that if we perturb each $V_{i}$ by adding a small affine function, then the minimum of these functions is generically stratified semiconcave. The proof will be based on a multi-transversality argument (Lemma 21).

In order to be clearer, we are going to first explain what happens in the case of the minimum of two functions $V_{1}$ and $V_{2}$. Consider two adjacent cubes $K_{1}$ and $K_{2}$ in $\Omega$ and let us prove how to move $V_{1}$ and $V_{2}$ in order to get that the minimum of $V_{1}$ and $V_{2}$ is stratified semiconcave on $\mathbb{R}^{N}$ (recall that $V_{1}$ and $V_{2}$ are quadratic functions defined on $\left.\mathbb{R}^{N}\right)$. Recall that by construction, for every $x \in \mathbb{R}^{N}$,

$$
\begin{aligned}
& V_{1}(x)=G_{1}(x)+\Psi_{1}(x)+\|x\|^{2}=\min _{i \in J^{1}}\left\{h_{i}^{1}(x)\right\}+\left(C_{1}+1\right)\|x\|^{2}, \\
& V_{2}(x)=G_{2}(x)+\Psi_{2}(x)+\|x\|^{2}=\min _{j \in J^{2}}\left\{h_{j}^{2}(x)\right\}+\left(C_{2}+1\right)\|x\|^{2} .
\end{aligned}
$$

If $C_{1}=C_{2}$ then the function $\min \left\{V_{1}, V_{2}\right\}$ is the sum of a piecewise affine semiconcave function and the function $x \mapsto\left(C_{1}+1\right)\|x\|^{2}$, so it is stratified semiconcave (by Proposition 19). Thus without loss of generality we can assume that $C_{1} \neq C_{2}$, assume for instance that $C_{2}=C_{1}+1$. Let $\beta=\left(\beta_{1}, \ldots, \beta_{\left|J^{2}\right|}\right)$ be a $\left|J^{2}\right|$-tuple of vectors in $\mathbb{R}^{N}$, define the function $V_{2}^{\beta}: \mathbb{R}^{N} \rightarrow \mathbb{R}$ by

$$
\forall x \in \mathbb{R}^{N}, \quad V_{2}^{\beta}(x):=\min _{j \in J^{2}}\left\{h_{j}^{2}(x)+\left\langle\beta_{j}, x\right\rangle\right\}+\left(C_{2}+1\right)\|x\|^{2},
$$

and define $V^{\beta}: \mathbb{R}^{n} \rightarrow \mathbb{R}$ by

$$
\forall x \in \mathbb{R}^{N}, \quad V^{\beta}(x):=\min \left\{V_{1}(x), V_{2}^{\beta}(x)\right\} .
$$

Let us prove that the function $V^{\beta}$ is generically stratified semiconcave on $\mathbb{R}^{N}$.

Lemma 21. The set of $\left(\beta_{1}, \ldots, \beta_{\left|J^{2}\right|}\right)$ for which the function $V^{\beta}$ is stratified semiconcave is an open dense set in $\left(\mathbb{R}^{N}\right)^{\left|J^{2}\right|}$.

Proof. First, note that if there exists a couple $(i, j) \in J^{1} \times J^{2}$ such that

$$
h_{i}^{1}(x)+\left(C_{1}+1\right)\|x\|^{2}=h_{j}^{2}(x)+\left\langle\beta_{j}, x\right\rangle+\left(C_{1}+2\right)\|x\|^{2},
$$

then this implies:

$$
\begin{aligned}
\|x\|^{2}-h_{i}^{1}(x)+h_{j}^{2}(x)+\left\langle\beta_{j}, x\right\rangle=0 & \Leftrightarrow\|x\|^{2}-\left\langle H_{i}^{1}, x\right\rangle+\left\langle H_{j}^{2}, x\right\rangle+\left\langle\beta_{j}, x\right\rangle=\hat{h}_{i}^{1}-\hat{h}_{j}^{2} \\
& \Leftrightarrow\|x\|^{2}+\left\langle-H_{i}^{1}+H_{j}^{2}+\beta_{j}, x\right\rangle=\hat{h}_{i}^{1}-\hat{h}_{j}^{2} .
\end{aligned}
$$

Thus we deduce that

$$
\left\|x-\frac{1}{2}\left(-H_{i}^{1}+H_{j}^{2}+\beta_{j}\right)\right\|^{2}=\hat{h}_{i}^{1}-\hat{h}_{j}^{2}+\frac{1}{4}\left\|-H_{i}^{1}+H_{j}^{2}+\beta_{j}\right\|^{2} .
$$

The function $V^{\beta}$ can be written as follows:

$$
V^{\beta}(x)=\min _{i \in J^{1}, j \in J^{2}}\left\{h_{i}^{1}(x)+\left(C_{1}+1\right)\|x\|^{2}, h_{j}^{2}(x)+\left\langle\beta_{j}, x\right\rangle+\left(C_{1}+2\right)\|x\|^{2}\right\}=\min _{i \in J^{1}, j \in J^{2}}\left\{E_{i}(x), E_{j}^{\prime}(x)\right\} .
$$


Study the cases where the minimum is attained by several $E_{i}$ and $E_{j}^{\prime}$ in the expression above. Let us prove that the set of points $x$ such that $E_{1}(x)=E_{j}^{\prime}(x)$ for every $j=1, \ldots, p$ (where $p$ is some integer in $\{1, \ldots, N\}$ ) is generically a sphere of dimension $N-p$ in $\mathbb{R}^{N}$. The other cases being similar, they are left to the reader.

Set

$$
\mathcal{O}:=\left\{x \in \mathbb{R}^{N}: E_{1}(x)=E_{j}^{\prime}(x), \forall j=1, \ldots, p\right\} .
$$

If $x$ belongs to $\mathcal{O}$, then by (26) it is in the intersection of $p$ spheres, that is:

$$
x \in \bigcap_{j=1, \ldots, p} S\left(z_{j}, \sqrt{R_{j}+\left\|z_{j}\right\|^{2}}\right),
$$

where $z_{j}:=\frac{1}{2}\left(-H_{1}^{1}+H_{j}^{2}+\beta_{j}\right)$ and $R_{j}:=\hat{h}_{1}^{1}-\hat{h}_{j}^{2}$. The conclusion (27) is equivalent to $x$ solving the system

$$
\left\{\begin{array}{l}
\|x\|^{2}-2\left\langle x, z_{1}\right\rangle=R_{1}, \\
\|x\|^{2}-2\left\langle x, z_{2}\right\rangle=R_{2}, \\
\vdots \\
\|x\|^{2}-2\left\langle x, z_{p}\right\rangle=R_{p} y
\end{array}\right.
$$

which can also be written as follows:

$$
\left\{\begin{array}{l}
\|x\|^{2}-2\left\langle x, z_{1}\right\rangle=R_{1}, \\
2\left\langle x, z_{1}-z_{2}\right\rangle=R_{2}-R_{1}, \\
\vdots \\
2\left\langle x, z_{1}-z_{p}\right\rangle=R_{p}-R_{1} .
\end{array}\right.
$$

This last system means that $x$ belongs to the intersection of the sphere centered at $z_{1}$ with radius $R_{1}$ and of the affine subspace $H_{\beta}$ of solution of the equation

$$
\left\{\begin{array}{l}
2\left\langle x, z_{1}-z_{2}\right\rangle=R_{2}-R_{1}, \\
2\left\langle x, z_{1}-z_{3}\right\rangle=R_{3}-R_{1}, \\
\vdots \\
2\left\langle x, z_{1}-z_{p}\right\rangle=R_{p}-R_{1} .
\end{array}\right.
$$

The affine subspace $H_{\beta}$ is of dimension $N-p+1$ if and only if the vectors $z_{1}-z_{2}, z_{1}-z_{3}, \ldots, z_{1}-z_{p}$ are linearly independent. Let $\Gamma:\left(\mathbb{R}^{N}\right)^{p} \rightarrow \mathbb{R}$ be the map which associates to each $p$-tuple $\left(\beta_{j}\right)_{j=1, \ldots, p}$ the sum of the squares of the determinants of the $(p-1) \times(p-1)$ matrices which are included in the rectangle matrix $M_{\beta}$ with columns $\left(z_{1}-z_{j}\right)_{j=2, \ldots, p}$. The rank of the family of vectors $\left(z_{1}-z_{j}\right)_{j=2, \ldots, p}$ equals $p-1$ if and only if there exists a square submatrix of $M_{\beta}$ (of order $p-1$ ) which is invertible. Hence by construction of $\Gamma$ :

$$
\operatorname{rank}\left\{\left(z_{1}-z_{j}\right)_{j=2, \ldots, p}\right\}=p-1 \Leftrightarrow \Gamma\left(\beta_{1}, \ldots, \beta_{p}\right) \neq 0 .
$$

The function $\Gamma$ is polynomial in the coordinates of the $\beta_{j}$ 's and moreover since $z_{1}-z_{j}=\frac{1}{2}\left(H_{1}^{2}-H_{j}^{2}+\beta_{1}-\beta_{j}\right)$, we have that

$$
\Gamma\left(-H_{1}^{2},-H_{2}^{2}-2 e_{1},-H_{3}^{2}-2 e_{2}, \ldots,-H_{p}^{2}-2 e_{p-1}\right)=1 .^{9}
$$

We conclude that the polynomial $\Gamma$ is not the zero polynomial, hence that the set

$$
T_{1}:=\left\{\left(\beta_{1}, \ldots, \beta_{p}\right) \in\left(\mathbb{R}^{N}\right)^{p}: \Gamma\left(\beta_{1}, \ldots, \beta_{p}\right) \neq 0\right\}
$$

is an open set with full measure in $\left(\mathbb{R}^{N}\right)^{p}$.

\footnotetext{
9 The family $\left(e_{1}, \ldots, e_{N}\right)$ denotes the usual basis of $\mathbb{R}^{N}$.
} 
Let us now study the intersection of the sphere $S\left(z_{1}, R_{1}\right)$ and of the affine subspace $H_{\beta}$. This intersection (whenever it is not empty) is a sphere of dimension $N-p$ if and only if the affine subspace $H_{\beta}$ is not tangent to the sphere, that is if

$$
d\left(z_{1}, H_{\beta}\right)^{2} \neq R_{1} .
$$

Let us evaluate this distance. If $P$ denotes the orthogonal projection of $z_{1}$ on $H_{\beta}$ then the distance equals $\left\|z_{1}-P\right\|^{2}$. Since $z_{1}-P$ is orthogonal to $H_{\beta}$, it is a linear combination of the vectors $z_{1}-z_{2}, \ldots, z_{1}-z_{p}$. Hence there exists $\alpha=\left(\alpha_{2}, \ldots, \alpha_{p}\right) \in \mathbb{R}^{p-1}$ such that

$$
z_{1}-P=\sum_{j=2}^{p} \alpha_{j}\left(z_{1}-z_{j}\right)
$$

On the other hand $P$ belongs to $H_{\beta}$ hence we have the following system:

$$
\left\{\begin{array}{l}
2\left\langle z_{1}-\sum_{j=2}^{p} \alpha_{j}\left(z_{1}-z_{j}\right), z_{1}-z_{2}\right\rangle=R_{2}-R_{1}, \\
2\left\langle z_{1}-\sum_{j=2}^{p} \alpha_{j}\left(z_{1}-z_{j}\right), z_{1}-z_{3}\right\rangle=R_{3}-R_{1}, \\
\vdots \\
2\left\langle z_{1}-\sum_{j=2}^{p} \alpha_{j}\left(z_{1}-z_{j}\right), z_{1}-z_{p}\right\rangle=R_{p}-R_{1} .
\end{array}\right.
$$

We recognize the system

$$
-2 M \alpha=T
$$

where $M$ is the Gram matrix

$$
M=\left(\begin{array}{ccc}
\left\langle z_{1}-z_{2}, z_{1}-z_{2}\right\rangle & \ldots & \left\langle z_{1}-z_{p}, z_{1}-z_{2}\right\rangle \\
\vdots & \ddots & \vdots \\
\left\langle z_{1}-z_{2}, z_{1}-z_{p}\right\rangle & \cdots & \left\langle z_{1}-z_{p}, z_{1}-z_{p}\right\rangle
\end{array}\right)
$$

and $T$ is the column vector

$$
\left(R_{2}-R_{1}-2\left\langle z_{1}, z_{1}-z_{2}\right\rangle, \ldots, R_{p}-R_{1}-2\left\langle z_{1}, z_{1}-z_{p}\right\rangle\right)^{t} .
$$

The matrix $M$ can be written as $M=M_{\beta}^{t} M_{\beta}$. (Recall that $M_{\beta}$ is the matrix whose the columns are the vectors $\left(z_{1}-z_{j}\right)_{j=2, \ldots, p}$.) Hence if the rank of $M_{\beta}$ equals $p-1$, then the determinant of the Gram matrix $M$ is different from zero (see [15, Theorem 1, p. 247]). In consequence the vector $\alpha$ is a solution of the Cramer system (32), that is

$$
\alpha=-\frac{1}{2} M^{-1} T
$$

Eq. (31) can be written as:

$$
\left\|\sum_{j=2}^{p} \alpha_{j}\left(z_{1}-z_{j}\right)\right\|^{2}-R_{1} \neq 0
$$

By (33) and from the definition of the $z_{j}$ 's, the vector $\operatorname{det}(M) \sum_{j=2}^{p} \alpha_{j}\left(z_{1}-z_{j}\right)$ depends affinly in the $\beta_{j}$ 's hence the quantity

$$
\operatorname{det}(M)^{2}\left[\left\|\sum_{j=2}^{p} \alpha_{j}\left(z_{1}-z_{j}\right)\right\|^{2}-R_{1}\right]
$$

is polynomial in the coordinates of the $\beta_{j}$ 's (and is not constant and equal to zero). We deduce that the set of $p$-tuples $\left(\beta_{1}, \ldots, \beta_{p}\right)$ such that $\Gamma\left(\beta_{1}, \ldots, \beta_{p}\right) \neq 0$ and such that $\left\|\sum_{j=2}^{p} \alpha_{j}\left(z_{1}-z_{j}\right)\right\|^{2}-R_{1} \neq 0$ is an open set 
of full measure in $\left(\mathbb{R}^{N}\right)^{p}$ (denote this set by $T_{2}$ ). This proves that the set $\mathcal{O}$ is generically a sphere of dimension $N-p$.

We leave it to the reader to convince himself that our proof shows that if the tuple $\left(\beta_{1}, \ldots, \beta_{\left|J^{2}\right|}\right)$ belongs to a generic set $T_{3}$ in $\left(\mathbb{R}^{N}\right)^{\left|J^{2}\right|}$, then for any subsets $I_{1}$ of $J^{1}$ and $I_{2}$ of $J^{2}$ such that $\left|I_{1}\right|+\left|I_{2}\right|-1 \leqslant N$, the set $\mathcal{O}_{I_{1}, I_{2}}$ of $x \in \mathbb{R}^{N}$ such that for any $i, j \in I_{1} \times I_{2}$

$$
E_{i}(x)=E_{j}^{\prime}(x),
$$

is a submanifold of $\mathbb{R}^{N}$ of dimension $N-\left|I_{1}\right|-\left|I_{2}\right|+1$.

Let us now prove that there exists a generic set $T_{4}$ in $\left(\mathbb{R}^{N}\right)^{\left|J^{2}\right|}$ such that if the tuple $\left(\beta_{1}, \ldots, \beta_{\left|J^{2}\right|}\right) \in T_{4}$ then for every $x \in \mathcal{O}_{I_{1}, I_{2}}$ with $\left|I_{1}\right|+\left|I_{2}\right|-1 \leqslant N$, the convex set $\partial V^{\beta}(x)$ has dimension $\left|I_{1}\right|+\left|I_{2}\right|-1$. Still once, in order to be clearer we assume that $I_{1}=\{1\}$ and that $I_{2}=\{1, \ldots, p\}$ with $p \leqslant N$.

Fix $x \in \mathcal{O}=\mathcal{O}_{I_{1}, I_{2}}$. Using the definitions of $H_{1}^{1}$ and $h_{j}^{2}$ for $j=1, \ldots, p$, we can write $\partial V^{\beta}(x)$ as follows:

$$
\partial V^{\beta}(x):=\operatorname{co}\left\{H_{1}^{1}+2 C_{1} x, H_{1}^{2}+\beta_{1}+2 C_{2} x, \ldots, H_{p}^{2}+\beta_{p}+2 C_{2} x\right\} .
$$

This set is a convex set of dimension $p$ if and only if the vectors $H_{1}^{2}+\beta_{1}-H_{1}^{1}+2 x, H_{2}^{2}+\beta_{2}-H_{1}^{1}+2 x, \ldots, H_{p}^{2}+$ $\beta_{p}-H_{1}^{1}+2 x$ are linearly independent (recall that $C_{2}-C_{1}=1$ ). By definition of the $z_{j}$ 's, this is equivalent to proving that the vectors $x-z_{1}, \ldots, x-z_{p}$ are linearly independent. We argue by contradiction. Assume that there exists $\alpha=\left(\alpha_{1}, \ldots, \alpha_{p}\right) \in \mathbb{R}^{p}$ such that

$$
\sum_{j=1}^{p} \alpha_{j}\left(x-z_{j}\right)=0
$$

Since $x$ belongs to $\mathcal{O}$, it satisfies Eqs. (30). Hence for every $k=2, \ldots, p$, the equation

$$
2\left(\sum_{j=1}^{p} \alpha_{j}\right)\left\langle x, z_{1}-z_{k}\right\rangle-2 \sum_{j=1}^{p} \alpha_{j}\left\langle z_{j}, z_{1}-z_{k}\right\rangle=0
$$

gives

$$
\left\{\begin{array}{l}
\left(\sum_{j=1}^{p} \alpha_{j}\right)\left(R_{2}-R_{1}\right)-2 \sum_{j=1}^{p} \alpha_{j}\left\langle z_{j}, z_{1}-z_{2}\right\rangle=0 \\
\left(\sum_{j=1}^{p} \alpha_{j}\right)\left(R_{3}-R_{1}\right)-2 \sum_{j=1}^{p} \alpha_{j}\left\langle z_{j}, z_{1}-z_{3}\right\rangle=0, \\
\vdots \\
\left(\sum_{j=1}^{p} \alpha_{j}\right)\left(R_{p}-R_{1}\right)-2 \sum_{j=1}^{p} \alpha_{j}\left\langle z_{j}, z_{1}-z_{p}\right\rangle=0 .
\end{array}\right.
$$

This means that the $p$-tuple $\left(\alpha_{1}, \ldots, \alpha_{p}\right)$ is solution of a system of $p-1$ linear equations in $\mathbb{R}^{p}$. We can write (36) with matrices; using the definition of the $z_{j}$ 's, the system is equivalent to

$$
\left(N+N^{\beta}\right) \alpha=0 \text {, }
$$

where the matrix $N$ is in $M_{p-1, p}(\mathbb{R})$ and does not depend upon the $\beta_{j}$ 's and where $N^{\beta} \in M_{p-1, p}(\mathbb{R})$ is defined by:

$$
N^{\beta}=\left(\begin{array}{cccc}
\left\langle\beta_{1}, \beta_{1}-\beta_{2}\right\rangle & \left\langle\beta_{2}, \beta_{1}-\beta_{2}\right\rangle & \ldots & \left\langle\beta_{p}, \beta_{1}-\beta_{2}\right\rangle \\
\left\langle\beta_{1}, \beta_{1}-\beta_{3}\right\rangle & \left\langle\beta_{2}, \beta_{1}-\beta_{3}\right\rangle & \ldots & \left\langle\beta_{p}, \beta_{1}-\beta_{3}\right\rangle \\
\vdots & \vdots & \ddots & \vdots \\
\left\langle\beta_{1}, \beta_{1}-\beta_{p}\right\rangle & \left\langle\beta_{2}, \beta_{1}-\beta_{p}\right\rangle & \ldots & \left\langle\beta_{p}, \beta_{1}-\beta_{p}\right\rangle
\end{array}\right) .
$$

This matrix can also be written as follows:

$$
N^{\beta}=\left(N_{1}^{t} M_{\beta}^{t}\right) M_{\beta}-\left(N_{2}^{t} M_{\beta}^{t}\right) M_{\beta},
$$


where the matrices $N_{1}, N_{2} \in M_{p, p-1}(\mathbb{R})$ are defined by,

$$
N_{1}:=\left(\begin{array}{cccc}
1 & 1 & \ldots & 1 \\
0 & 0 & \ldots & 0 \\
\vdots & \vdots & \ddots & \vdots \\
0 & \ldots & \ldots & 0
\end{array}\right)
$$

and

$$
N_{2}:=\left(\begin{array}{ccccc}
0 & 0 & \ldots & \ldots & 0 \\
1 & 0 & \ldots & \ldots & 0 \\
0 & 1 & \ddots & \ldots & \vdots \\
\vdots & 0 & \ddots & \ddots & \vdots \\
\vdots & \vdots & \ddots & \ddots & 0 \\
0 & \ldots & \ldots & 0 & 1
\end{array}\right)
$$

The system (37) becomes

$$
\left[N+\left(N_{1}-N_{2}\right)^{t} M_{\beta}^{t} M_{\beta}\right] \alpha=0 .
$$

Now if $\left(\beta_{1}, \ldots, \beta_{p}\right)=\left(\lambda e_{1}, \ldots, \lambda e_{p}\right)$ where $\lambda$ is some real number, then we deduce that $\alpha$ is solution of the system

$$
\left[N+\lambda^{2}\left(N_{1}-N_{2}\right)^{t}\right] \alpha=0 .
$$

Note that the $p-1$ last columns of the matrix $\left(N_{1}-N_{2}\right)^{t}$ is a matrix in $M_{p-1, p-1}(\mathbb{R})$ which equals $-I_{p-1}$. Hence we deduce that the matrix $Q(\lambda) \in M_{p-1, p-1}(\mathbb{R})$ which corresponds to the $p-1$ last columns of the matrix $[N+$ $\left.\lambda^{2}\left(N_{1}-N_{2}\right)^{t}\right]$ has rank $p-1$ for almost every $\lambda \in \mathbb{R}$. This proves that the matrix $Q\left(\beta_{1}, \ldots, \beta_{p}\right) \in M_{p-1, p-1}(\mathbb{R})$ which equals the $p-1$ last columns of $\left[N+\left(N_{1}-N_{2}\right)^{t} M_{\beta}^{t} M_{\beta}\right]$ has rank $p-1$ for $\left(\beta_{1}, \ldots, \beta_{p}\right)$ in an open dense set of full measure $T \subset\left(\mathbb{R}^{N}\right)^{p}$. For such $p$-tuples $\left(\beta_{1}, \ldots, \beta_{p}\right)$, the set of solutions of (36) is a vector line in $\mathbb{R}^{p}$. In fact we can give a nonzero vector $\left(\alpha_{1}, \ldots, \alpha_{p}\right)$ which spans this vector line. For instance it is the solution $\left(\overline{\alpha_{1}}, \ldots, \overline{\alpha_{p}}\right)$ of the linear system (39) with first coefficient

$$
\overline{\alpha_{1}}\left(\beta_{1}, \ldots, \beta_{p}\right)=\operatorname{det}\left(Q\left(\beta_{1}, \ldots, \beta_{p}\right)\right) \neq 0 .
$$

Hence by Cramer's formulas the vector $\left(\overline{\alpha_{1}}, \ldots, \overline{\alpha_{p}}\right)$ is a polynomial in the $\beta_{j}^{\prime} s$. Moreover whenever $\left(\beta_{1}, \ldots, \beta_{p}\right)=$ $\left(\lambda e_{1}, \ldots, \lambda e_{p}\right)$, each $\overline{\alpha_{j}}(j=1, \ldots, p)$ is a polynomial in $\lambda$ of degree $2(p-1)$ with leading term 1 .

On the other hand, (35) also implies that

$$
\left\|\sum_{j=1}^{p} \alpha_{j}\left(x-z_{j}\right)\right\|^{2}=0 .
$$

Hence we get that

$$
\sum_{j=1}^{p} \alpha_{j}^{2}\left\|x-z_{j}\right\|^{2}+\sum_{j \neq k} 2 \alpha_{j} \alpha_{k}\left\langle x-z_{j}, x-z_{k}\right\rangle=0 .
$$

But for each couple $(j, k) \in\{1, \ldots, p\}^{2},(28)$ gives that

$$
2\left\langle x-z_{j}, x-z_{k}\right\rangle=2\left\|x-z_{j}\right\|^{2}+2\left\langle x-z_{j}, z_{j}-z_{k}\right\rangle=2\left\|x-z_{j}\right\|^{2}+R_{k}-R_{j}-2\left\langle z_{j}, z_{j}-z_{k}\right\rangle .
$$

By (27), we know that for every $j,\left\|x-z_{j}\right\|^{2}=R_{j}+\left\|z_{j}\right\|^{2}$. Thus (40) becomes 


$$
\begin{aligned}
& \sum_{j=1}^{p} \alpha_{j}^{2}\left(R_{j}+\left\|z_{j}\right\|^{2}\right)+\sum_{j \neq k} \alpha_{j} \alpha_{k}\left[2\left(R_{j}+\left\|z_{j}\right\|^{2}\right)+R_{k}-R_{j}-2\left\langle z_{j}, z_{j}-z_{k}\right\rangle\right] \\
& \quad=\sum_{j=1}^{p} \alpha_{j}^{2}\left(R_{j}+\left\|z_{j}\right\|^{2}\right)+\sum_{j \neq k} \alpha_{j} \alpha_{k}\left[R_{j}+R_{k}+2\left\langle z_{j}, z_{k}\right\rangle\right]=0 .
\end{aligned}
$$

Denote by $P\left(\alpha_{1}, \ldots, \alpha_{p}\right)$ the polynomial in several variables $\alpha_{1}, \ldots, \alpha_{p}$ which appears in (41). Let us prove that whenever $\left(\beta_{1}, \ldots, \beta_{p}\right) \in T$, the vector $\left(\overline{\alpha_{1}}, \ldots, \overline{\alpha_{p}}\right)$ is generically not a solution of (41).

Actually, the function $P \circ\left(\overline{\alpha_{1}}, \ldots, \overline{\alpha_{p}}\right)$ is a polynomial in the coefficient of the $\beta_{j}$ 's. But whenever $\left(\beta_{1}, \ldots, \beta_{p}\right)=\left(\lambda e_{1}, \ldots, \lambda e_{p}\right) \in T$, the function $P \circ \bar{\alpha}(\lambda)$ is a polynomial in $\lambda$ of degree $2(2 p-1)$ with leading term $p$ hence $P \circ \bar{\alpha}(\lambda) \neq 0$ for almost every $\lambda \in \mathbb{R}$. Since the polynomial $P$ is homogeneous, this proves that the intersection of the set of solutions of (41) and of the solution of (36) equals generically $\{0\}$. This proves that for every $x \in \mathcal{O}$, the vectors $x-z_{1}, \ldots, x-z_{p}$ are independent and hence that $\partial V^{\beta}(x)$ is a convex set of dimension $p$ in $\mathbb{R}^{N} .10$

We leave it to the reader to develop this proof for any subset $I_{1}$ and $I_{2}$ of $J^{1}$ and $J^{2}$. In conclusion, we proved that there exists a generic set $T_{4}$ in $\left(\mathbb{R}^{N}\right)^{\left|J^{2}\right|}$ such that if $\left(\beta_{1}, \ldots, \beta_{\left|J^{2}\right|}\right) \in T_{4}$ then for any $x \in \mathcal{O}_{I_{1}, I_{2}}$, the generalized gradient of $V^{\beta}$ has dimension $\min \left\{N,\left|I_{1}\right|+\left|I_{2}\right|-1\right\}$ and has $\min \left\{N,\left|I_{1}\right|+\left|I_{2}\right|-1\right\}+1$ extreme points.

In conclusion, we proved that if $\left(\beta_{1}, \ldots, \beta_{\left|J^{2}\right|}\right)$ belongs to the generic set $T_{3} \cap T_{4} \subset\left(\mathbb{R}^{N}\right)^{\left|J^{2}\right|}$, then the set $\mathcal{O}_{I_{1}, I_{2}}$ is a submanifold of $\mathbb{R}^{N}$ of dimension $\max \left\{0, N-\left|I_{1}\right|-\left|I_{2}\right|+1\right\}$ and that the generalized gradient of $V^{\beta}$ has dimension $\min \left\{N,\left|I_{1}\right|+\left|I_{2}\right|-1\right\}$ and has $\min \left\{N,\left|I_{1}\right|+\left|I_{2}\right|-1\right\}+1$ extreme points. Furthermore it is clear from the proof that the closure of each of the $\mathcal{O}_{I_{1}, I_{2}}$ 's is a smooth submanifold and that the function $V^{\beta}$ is smooth on it. In addition, it is clear by (34) that the functions which give the extreme points of $\partial V^{\beta}$ are smooth and can be smoothly extended to the closure of each connected component of $\mathcal{O}_{I_{1}, I_{2}}$ (and that $\partial V^{\beta}$ is smoothly extended into a convex set with the right number of extreme points by footnote 9). Thus this proves that assertions (ii), (iii) and (iv) in Definition 18 hold. Finally, by classical properties of semiconcave function (see Appendix), we have that for any $x \in \mathcal{O}_{I_{1}, I_{2}}$,

$$
T_{x} \mathcal{O}_{I_{1}, I_{2}}=\partial V^{\beta}(x)^{\perp} .
$$

Since the multivalued map $\partial V^{\beta}(\cdot)$ is upper semicontinuous, we deduce from the facts above that $\Sigma\left(V^{\beta}\right)$ is generically a Whitney stratification. This completes the proof of Lemma 21.

We proved that the minimum of both functions $V_{1}$ and $V_{2}$ can in fact be approximated generically by a stratified semiconcave function $V^{\beta}$. Since the covering given in $(P 1)$ is locally finite, starting from $V_{1}$, we will are able to construct step by step some functions $V_{2}^{\beta}, \ldots, V_{l}^{\beta}$ such that the function $\min \left\{V_{1}, \ldots, V_{2}^{\beta}, \ldots, V_{l}^{\beta}\right\}$ is stratified semiconcave in $\mathbb{R}^{N}$. But when we have constructed the $V_{l}^{\beta}$,s on all the adjacent cubes to $K_{1}$, the next construction will not change the global minimum function on $K_{1}$. In this way we perform the construction of a global stratified semiconcave on $\Omega{ }^{11}$ Furthermore it is clear that since the initial function $\widetilde{V}$ satisfies (17) and (18), if the perturbations $\beta$ are taken small enough, then the resulting stratified semiconcave (on $\Omega$ ) function $V$ satisfies (17) and (18) for the function $2 \epsilon(\cdot)$. This concludes the proof of Theorem 20 .

\footnotetext{
10 Notice that actually we also proved that for each $x \in \overline{\mathcal{O}}$, the set defined by the right-hand side in (34) is a convex compact set of dimension $p$ in $\mathbb{R}^{N}$ (because any $x \in \mathcal{O}$ satisfies (30)).

11 Notice that the function that we obtain is not stratified at the origin because the stratification is not locally finite in the neighborhood of 0 .
} 


\subsection{Additional comments}

In fact, the procedure of regularization that we apply in the previous section can be used for any semiconcave function. We leave as an exercise for the reader to supply the details of the proof of the following theorem:

Theorem 22. Let $\Omega$ be an open set of $\mathbb{R}^{N}$. If $u: \Omega \rightarrow \mathbb{R}$ is a semiconcave function then for any continuous function $\epsilon: \Omega \rightarrow(0, \infty)$ such that for every $x \in \Omega, B_{N}(x, \epsilon(x)) \subset \Omega$, there exists a stratified semiconcave function $u_{\epsilon}$ such that for any $x \in \Omega$,

(i) $\left|u(x)-u_{\epsilon}(x)\right| \leqslant \epsilon(x)$,

(ii) $\partial_{P} u_{\epsilon}(x) \subset \partial_{P} u\left(x+\epsilon(x) B_{N}\right)+\epsilon(x) B_{N}$.

This result applies to the approximation of viscosity solutions introduced by Crandall and Lions [17,14]. If $F: \Omega \times \mathbb{R} \times \mathbb{R} \rightarrow \mathbb{R}$ denotes a Hamiltonian which is continuous in the three variables, Theorem 22 gives the following:

Corollary 23. Let $\Omega$ be an open set of $\mathbb{R}^{N}$. If $u: \Omega \rightarrow \mathbb{R}$ is a semiconcave supersolution of the Hamilton-Jacobi equation

$$
F(x, u, D u)=0,
$$

then for any continuous function $\epsilon: \Omega \rightarrow(0, \infty)$, there exists a function $v: \Omega \rightarrow \mathbb{R}$ such that

(i) $v$ is a supersolution of $F(x, v, D v)-\epsilon(x)=0$,

(ii) the function $v$ is stratified semiconcave,

(iii) $|u-v| \leqslant \epsilon$.

Proof. Recall that since the Hamiltonian $F$ is continuous in the third variable then the supersolution $u$ of (42) satisfies

$$
\forall x \in \Omega, \forall \zeta \in \partial_{L} u(x), \quad F(x, u(x), \zeta) \geqslant 0 .
$$

By semiconcavity of $u$, for every $x \in \Omega$, the limiting subgradient $\partial_{L} u(x)$ is a compact subset of $\mathbb{R}^{N}$. Hence the continuity of the Hamiltonian $F$ in the last variable implies that there exists a positive constant $\delta_{x}$ such that if $\zeta \in \partial_{L} u(x)+2 \delta_{x} B_{N}$ then

$$
F(x, u(x), \zeta) \geqslant-\frac{\epsilon(x)}{2} \text {. }
$$

But the limiting subgradient is an upper semicontinuous multivalued map, hence there exists $\mu_{x}>0$ such that if $y \in x+\mu_{x} B_{N}$ then

$$
\partial_{L} u(y) \subset \partial_{L} u(x)+\delta_{x} B_{N} .
$$

In addition the continuity of $F$ in the second variable and the compactness of the limiting subgradients imply the existence of $\beta_{x}>0$ such that if $\left|u^{\prime}-u(x)\right| \leqslant \beta_{x}$ then

$$
\forall \zeta \in \partial_{L} u(x)+\delta_{x} B_{N}, \quad\left|F\left(x, u^{\prime}, \zeta\right)-F(x, u(x), \zeta)\right| \leqslant \frac{\epsilon(x)}{2} .
$$

Set for every $x \in \Omega$,

$$
\epsilon^{\prime}(x):=\min \left\{\epsilon(x), \frac{d\left(x, \Omega^{c}\right)}{2}, \inf _{y \in \mathbb{R}^{N}}\left\{\min \left\{\mu_{x}, \beta_{x}\right\}\right\}\right\} .
$$


The three functions that appear in the minimum above are continuous, hence $\epsilon^{\prime}$ is continuous. Since for any $x \in$ $\Omega, \epsilon^{\prime}(x) \leqslant d\left(x, \Omega^{c}\right) / 2$, we can apply Theorem 22 . We get the existence of a stratified semiconcave function $v: \Omega \rightarrow \mathbb{R}$ with

$$
\forall x \in \Omega, \quad|u(x)-v(x)| \leqslant \epsilon^{\prime}(x)
$$

and

$$
\forall x \in \Omega, \quad \partial_{P} v(x) \subset \partial_{P} u\left(x+\epsilon^{\prime}(x) B_{N}\right)+\epsilon^{\prime}(x) B_{N} .
$$

Assertions (ii) and (iii) are already given, it remains to prove (i).

Consider $x \in \Omega$ and $\zeta \in \partial_{P} v(x)$. The definition of $\epsilon^{\prime}(x)$ combined with (47) and (44) gives that

$$
\zeta \in \partial_{P} u\left(x+\mu_{x}(x) B_{N}\right)+\delta_{x} B_{N} \subset \partial_{L} u(x)+2 \delta_{x} B_{N} .
$$

Hence by (43) we deduce that $F(x, u(x), \zeta) \geqslant-\frac{\epsilon(x)}{2}$. But since $\epsilon^{\prime}(x) \leqslant \beta_{x}$, (46) with (45) imply that

$$
F(x, v(x), \zeta) \geqslant-\epsilon(x) .
$$

We conclude easily.

\section{Consequences for stabilizing feedbacks}

\subsection{Carathéodory stabilizing feedbacks}

In our previous paper [24], we proved that every GAC control system of the form (1) (we authorized in fact control systems with drift as well) admits a feedback $\alpha(\cdot): \mathbb{R}^{N} \rightarrow \overline{B_{m}}$ which makes the closed-loop system (2) GAS in the sense of Carathéodory. ${ }^{12}$ In order to produce such a feedback, we considered a semiconcave controlLyapunov function and we constructed a continuous feedback outside a subset of its singular set. Although this technique achieved the construction of a stabilizing feedback in the sense of Carathéodory, we were not able to describe the behavior of the closed-loop system around the singular set and then to deduce the existence of a AGAS feedback. Here, in our situation, the knowledge of a stratified semiconcave CLF will help us to construct explicitly the stabilizing feedback in a neighborhood of "each" stratum of the singular set $\Sigma(V)$.

Set $\Omega:=\mathbb{R}^{N} \backslash\{0\}$. Let us consider $V$ to be a stratified semiconcave control-Lyapunov function for the control system (1). Before proving Theorem 5, we need to construct some stabilizing feedback $\alpha(\cdot): \mathbb{R}^{N} \rightarrow \overline{B_{m}}$ for which we understand exactly the bifurcation points which are produced by the closed-loop system (2) (which stabilizes in the sense of Carathéodory); this particular result will be precisely stated in Theorem 37 . Let us describe the construction of the feedback $\alpha(\cdot)$. In a first time, we do not assume that $N=2$.

First of all in order to simplify our construction, we modify the control-Lyapunov function $V$. We claim the following:

Lemma 24. There exists some function $W: \Omega \rightarrow \mathbb{R}$ which is stratified semiconcave on $\Omega$, proper, which satisfies $\lim _{x \rightarrow 0} W(x)=-\infty$ and such that

$$
\forall x \in \Omega, \forall \zeta \in \partial_{L} W(x), \quad \min _{\alpha \in \overline{B_{m}}}\{\langle f(x, \alpha), \zeta\rangle\} \leqslant-1 .
$$

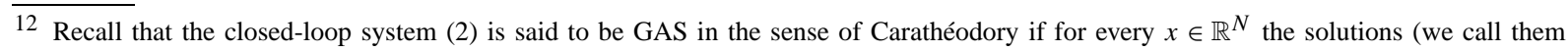
Carathéodory solutions) of

$$
\dot{x}(t)=f(x(t), \alpha(x(t))) \text { a.e., } \quad x(0)=x_{0},
$$

exist, converge to the origin as $t \rightarrow \infty$ and satisfy the property of Lyapunov stability.
} 
Proof. Set for every $x \in \Omega, W(x):=\ln V(x)$. By smoothness of the logarithm, we have for every $x \in \Omega$,

$$
\partial W(x)=\frac{1}{V(x)} \partial V(x) .
$$

Consequently, since the function $x \mapsto \ln x$ is smooth, concave and increasing on $[0, \infty)$ it is straightforward to show that $W$ is stratified semiconcave on $\Omega$, proper, satisfies $\lim _{x \rightarrow 0} W(x)=-\infty$ and such that

$$
\forall x \in \Omega, \forall \zeta \in \partial_{P} W(x), \quad \min _{\alpha \in \overline{B_{m}}}\{\langle f(x, \alpha), \zeta\rangle\} \leqslant-1 .
$$

By continuity of the dynamics $f$ the same property holds for limiting subgradients. Therefore we get (48).

Define the function $\Psi: \Omega \rightarrow(-\infty, 0]$ as follows,

$$
\forall x \in \Omega, \quad \Psi(x):=\min _{\alpha \in \overline{B_{m}}} \max _{\zeta \in \partial W(x)}\{\langle f(x, \alpha), \zeta\rangle\} .
$$

Since for any $x \in \Omega, f(x, 0)=0$ we verify that the function $\Psi$ has nonpositive values; moreover we have the following result:

Lemma 25. The function $\Psi$ is upper semicontinuous on $\Omega$.

Proof. Since for any $x \in \Omega$, the sets $\partial W(x)$ and $\overline{B_{m}}$ are convex and since the functions $\zeta \mapsto\langle f(x, \alpha), \zeta\rangle$ and $\alpha \mapsto\langle f(x, \alpha), \zeta\rangle$ are affine (do not forget that $f(x, \alpha)=\sum_{i=1}^{m} \alpha_{i} f_{i}(x)$ ), the Minimax Theorem (see [5, Theorem 3.7.9, p. 115]) allows us to write the function $\Psi$ as follows:

$$
\begin{aligned}
& \forall x \in \Omega, \quad \Psi(x)=\max _{\zeta \in \partial W(x)} \min _{\alpha \in \overline{B_{m}}}\{\langle f(x, \alpha), \zeta\rangle\}=\max _{\zeta \in \partial W(x)} \min _{\alpha \in \overline{B_{m}}}\left\{\left\langle\sum_{i=1}^{m} \alpha_{i} f_{i}(x), \zeta\right\rangle\right\} \\
& =\max _{\zeta \in \partial W(x)} \min _{\alpha \in \overline{B_{m}}}\left\{\sum_{i=1}^{m} \alpha_{i}\left\langle f_{i}(x), \zeta\right\rangle\right\} .
\end{aligned}
$$

Now since for every $\zeta \in R^{N}$ the map

$$
x \mapsto \min _{\alpha \in \overline{B_{m}}}\left\{\sum_{i=1}^{m} \alpha_{i}\left\langle f_{i}(x), \zeta\right\rangle\right\}
$$

is continuous, the upper semicontinuity of the multivalued map $x \mapsto \partial W(x)$ gives the result.

Since the function $W$ is stratified semiconcave, its singular set can be written as a countable disjoint union of strata of dimension less than $N$. Actually each singular subset $\Sigma^{k}(W)$ (for $k \in\{1, \ldots, N\}$ ) is a locally finite disjoint union of strata of dimension $N-k$, hence there exists $N$ countable sets $I_{1}, \ldots, I_{N}$ such that

$$
\Sigma(W)=\bigcup_{k \in\{1, \ldots, N\}} \Sigma^{k}(W)=\bigcup_{k \in\{1, N\}} \bigcup_{i \in I_{k}} S_{i}^{N-k} .
$$

From Definition 18 we note that the function $\Psi$ is smooth on each stratum of $\Sigma(W)$; as a consequence it will enjoy the following property:

Lemma 26. For almost every $\lambda \in(0,1)$, the following property is satisfied:

For each $k \in\{1, \ldots, N\}$, for any $i \in I_{k}$ either, the set $\left\{x \in S_{i}^{N-k}: \Psi(x)=-\lambda\right\}$ is empty or a Lipschitz submanifold of $S_{i}^{N-k}$ of dimension $N-k-1$ (if $k=N$ this means that the set is empty). 
Proof. By (50) the function $\Psi$ can be expressed as

$$
\Psi(x)=\max _{\zeta \in \partial W(x)} \min _{\alpha \in \overline{B_{m}}}\left\{\sum_{i=1}^{m} \alpha_{i}\left\langle f_{i}(x), \zeta\right\rangle\right\} .
$$

But by the Cauchy-Schwarz Theorem the minimum inside this formula can be computed, and therefore we have that for every $x \in \Omega$,

$$
\Psi(x)=\max _{\zeta \in \partial W(x)}\left\{-\sqrt{\sum_{i=1}^{m}\left\langle f_{i}(x), \zeta\right\rangle^{2}}\right\} .
$$

We are now ready to prove Lemma 26. In fact it is sufficient to prove that for every integer $p \geqslant 2$, for almost every $\lambda \in(0,1-1 / p)$, the property given in the lemma is satisfied. Fix $p \in \mathbb{N} \backslash\{1\}$ and let us prove this fact.

Let $S_{i}^{N-k}$ be a stratum of dimension $N-k$ (with $k \in\{1, \ldots, N-1\}$ and $i \in I_{k}$ ) in the Whitney stratification of $\Sigma(W)$. By Definition 18 there exist $k+1$ functions $\zeta_{1}, \ldots, \zeta_{k+1}$ which are smooth on $S$ and such that for every $x \in S$,

$$
\partial W(x)=\operatorname{co}\left\{\zeta_{1}(x), \ldots, \zeta_{k+1}(x)\right\} .
$$

If we denote by $\Delta_{k+1}$ the simplex of dimension $k+1$ defined by

$$
\Delta_{k+1}:=\left\{\left(t_{1}, \ldots, t_{k+1}\right) \in \mathbb{R}^{k+1}: \forall j, t_{j} \geqslant 0 \text { and } \sum_{i=1}^{k+1} t_{i}=1\right\},
$$

this means that for every $x \in S_{i}^{N-k}$ we have,

$$
\Psi(x)=\max _{t \in \Delta_{k+1}}\left\{-\sqrt{\sum_{j=1}^{k+1} \sum_{i=1}^{m} t_{j}\left\langle f_{i}(x), \zeta_{j}(x)\right\rangle^{2}}\right\} .
$$

Note that for every $x \in S_{i}^{N-k}$ the limiting gradient of $W$ at $x$ equals the set $\left\{\zeta_{1}(x), \ldots, \zeta_{k+1}(x)\right\}$. Thus by (48) this implies that for every $j \in\{1, \ldots, k+1\}$ we have

$$
-\sqrt{\sum_{i=1}^{m}\left\langle f_{i}(x),\left.\zeta_{j}(x)\right|^{2}\right.} \leqslant-1 .
$$

Denote by $\mathcal{S}$ the set of $x \in S_{i}^{N-k}$ such that $-\Psi(x) \in(0,1-1 / p)$; of course $\mathcal{S}$ is open in $S_{i}^{N-k}$.

From (52) we deduce that for each point $x \in \mathcal{S}$, the maximum in (51) cannot be attained at some $(k+1)$-tuples of the form $(0, \ldots, 0,1,0, \ldots, 0)$ i.e. at some vertex of the simplex $\Delta_{k+1}$. Actually since by Definition 18 the maps $\zeta_{1}, \ldots, \zeta_{k+1}$ can be smoothly extended to $\overline{S_{i}^{N-k}}$, this means that for each $j \in\{1, \ldots, k+1\}$ there exists some neighborhood $\mathcal{V}_{j}$ of the $\left(k+1\right.$ )-tuple $t^{j}$ (with $t_{j}=1$ and $t_{l}=0$ for $l \neq j$ ), such that for any $x \in \mathcal{S}$ the maximum in (51) is not attained in $\bigcup_{j=1}^{k+1} \mathcal{V}_{j}$. In consequence, there exists a smooth compact submanifold $M_{k, p}$ with boundary in the simplex $\Delta_{k+1}$ such that for any $x \in \mathcal{S}$,

$$
\Psi(x)=\max _{t \in M_{k, p}}\left\{-\sqrt{\sum_{j=1}^{k+1} \sum_{i=1}^{m} t_{j}\left\langle f_{i}(x), \zeta_{j}(x)\right\rangle^{2}}\right\} .
$$

To summarize, we have shown that on the open set $\mathcal{S}$, the function $\Psi$ can represented as the maximum of several smooth functions over a compact smooth manifold with boundary. Therefore the Morse-Sard theorem developed 
in [26, Theorem 3] applies, ${ }^{13}$ then we get that for almost every $\lambda \in(0,1-1 / p)$ the set $\{x \in \mathcal{S}: \Psi(x)=-\lambda\}$ is a Lipschitz submanifold of $S_{i}^{N-k}$ of codimension 1 .

We proved the property of Lemma 26 for every stratum of dimension $1, \ldots, N-1$. On the other hand the result is obvious for strata of dimension zero. Finally, the global number of strata of $\Sigma(W)$ being countable, we conclude easily.

Let $\lambda \in(0,1)$ be some constant satisfying the property of Lemma 26 . For each $k \in\{1, \ldots, N-1\}$, we divide the set $\Sigma^{k}(W)$ into three subsets as follows: Let $S$ be some stratum in $\Sigma^{k}(W)$,

- we say that $S$ is stable if for every $x \in S, \Psi(x) \leqslant-\lambda$;

- we say that $S$ is repulsive if for every $x \in \bar{S}, \Psi(x)>-\lambda$;

- we say that $S$ is a bifurcation stratum if there exists $x \in \bar{S}$ and $y \in S$ such that $\Psi(x) \leqslant-\lambda$ and $\Psi(y)>-\lambda$.

We denote by $\Sigma_{s}^{k}(W)$ (resp. by $\Sigma_{r}^{k}(W)$ and resp. by $\left.\Sigma_{b}^{k}(W)\right)$ the set of stable (resp. repulsive and resp. bifurcation) strata of $\Sigma^{k}(W)$. Thus the singular set $\Sigma(W)$ is partitioned into three subsets:

$$
\Sigma(W)=\Sigma_{s}(W) \cup \Sigma_{r}(W) \cup \Sigma_{b}(W) .
$$

Recall that for each stratum $S$ in $\Sigma_{b}(W)$, the set

$$
\{x \in S: \Psi(x)=-\lambda\}
$$

is a (nonempty) Lipschitz submanifold of $S$ of codimension 1. Hence whenever $S$ is a bifurcation stratum we can define the Lipschitz manifold (with boundary) $\widetilde{S}$ by

$$
\widetilde{S}:=\{x \in \bar{S}: \Psi(x) \geqslant-\lambda\} .
$$

We define the set $\mathcal{S} \subset \Sigma(W)$ by:

$$
\mathcal{S}:=\Sigma_{r}(W) \cup \bigcup_{S \in \Sigma_{b}(W)} \tilde{S} .
$$

This set satisfies the following:

\section{Lemma 27. The set $\mathcal{S}$ is closed in $\Omega$.}

Proof. Both sets $\Sigma_{r}(W)$ and $\Sigma_{b}(W)$ are locally finite unions of strata of the singular set $\Sigma(W)$. From definition of repulsive strata, this implies that the set $\Sigma_{r}(W)$ is closed in $\Omega$. On the other hand, by upper semicontinuity of the function $\Psi$ each stratum $\widetilde{S}$ given by (53) is closed. Hence we deduce that the set $\mathcal{S}$ is closed.

Therefore the subset of $\Omega$ defined by

$$
\mathcal{D}:=\Omega \backslash \mathcal{S}
$$

$\overline{13}$ Theorem 3 in [26] asserts the following:

Let $\mathcal{U}$ be an open subset of $\mathbb{R}^{n}$ and let $N$ be a smooth compact manifold. Let $\phi: \mathcal{U} \times N \rightarrow \mathbb{R}$ be a smooth function. Then the function $f: \mathcal{U} \rightarrow \mathbb{R}$ defined by

$$
f(x):=\min _{q \in N}\{\phi(x, q)\}
$$

satisfies the Morse-Sard theorem: That is, for almost every $\lambda$ in $f(\mathcal{U})$ the set $\{x \in \mathcal{U}: f(x)=\lambda\}$ is a Lipschitz submanifold of $\mathcal{U}$ of codimension one. In particular the result applies also in the case of smooth compact manifolds with boundary. 
is an open dense set of $\mathbb{R}^{N}$. From now we follow more or less the method of proof that we used in [24] in order to produce a stabilizing feedback which was continous on an open dense set. First we define a multivalued map $G_{1}: \mathcal{D} \rightarrow \overline{B_{m}}$ as follows:

For every $x \in \mathcal{D}$, we set

$$
G_{1}(x):=\left\{\alpha \in \overline{B_{m}}: \forall \zeta \in \partial W(x),\langle f(x, \alpha), \zeta\rangle \leqslant-\lambda\right\} .
$$

It enjoys the following property:

Lemma 28. The multifunction $G_{1}$ has nonempty compact convex values and is lower semicontinuous on the set $\mathcal{D}$.

Proof. By construction, we have that for every $x \in \mathcal{D}, \Psi(x) \leqslant-\lambda$. Hence by (49), this means that the set $G_{1}(x)$ is never empty on $\mathcal{D}$. On the other hand, recall that for every $x \in \mathcal{D}$ and for every $\zeta \in \partial W(x)$,

$$
\langle f(x, \alpha), \zeta\rangle=\sum_{i=1}^{m} \alpha_{i}\left\langle f_{i}(x), \zeta\right\rangle .
$$

Hence it is clear that for every $x \in \mathcal{D}$, the set $G_{1}(x)$ is compact and convex. Let us prove the upper semicontinuity of $G_{1}$. We then have to prove that for any sequence $\left(x_{n}\right)_{n}$ of points in $\mathcal{D}$ converging to some $x \in \mathcal{D}$, and for any $\alpha \in G_{1}(x)$, there exists a sequence $\left(\alpha_{n}\right)_{n}$ of points in $G_{1}\left(x_{n}\right)$ with limit $\alpha$.

Let $\left(x_{n}\right)_{n}$ be a sequence in $\mathcal{D}$ converging to $\bar{x} \in \mathcal{D}$, and let $\bar{\alpha} \in G_{1}(\bar{x})$. Define for every $x \in \mathcal{D}$ the function $g_{x}: \mathbb{R}^{m} \rightarrow \mathbb{R}$ by

$$
\forall \alpha \in \mathbb{R}^{m}, \quad g_{x}(\alpha):=\max _{\zeta \in \partial W(x)}\langle f(x, \alpha), \zeta\rangle .
$$

The function $g_{x}$ is convex as a maximum of affine functions. We claim that for each integer $n$, there exists $\alpha_{n} \in$ $G_{1}\left(x_{n}\right)$ such that

$$
\left\|\bar{\alpha}-\alpha_{n}\right\| \leqslant 2 \sqrt{1-\frac{g_{x_{n}}(\bar{\alpha})^{2}}{\lambda^{2}}} .
$$

We argue by contradiction; assume that for some integer $n$ we have

$$
\forall \alpha \in G_{1}\left(x_{n}\right), \quad\|\bar{\alpha}-\alpha\|>2 \sqrt{1-\frac{g_{x_{n}}(\bar{\alpha})^{2}}{\lambda^{2}}}=: l .
$$

In particular this implies that $\bar{\alpha} \notin G_{1}\left(x_{n}\right)$ and that $g_{x_{n}}(\bar{\alpha})>-\lambda$ (in fact by upper semicontinuity of the multivalued map $x \mapsto \partial W(x)$, we can assume that $g_{x_{n}}(\bar{\alpha}) \in(-\lambda, 0)$ ). In addition, since $g_{x_{n}}(0)=0$ and $G_{1}\left(x_{n}\right) \neq \emptyset$, (56) means that there exists $\alpha \in G_{1}\left(x_{n}\right)$ with $g_{x_{n}}(\alpha)=-\lambda$ and such that

$$
\|\bar{\alpha}-\alpha\|>l .
$$

By Pythagorean Theorem, this implies that if we set $\beta:=\frac{\bar{\alpha}+\alpha}{2} \in \overline{B_{m}}$, we have

$$
\|\beta\|<\sqrt{1-\frac{l^{2}}{4}} .
$$

Note that by convexity of the function $g_{x_{n}}$, we have that $g_{x_{n}}(\beta) \leqslant g_{x_{n}}(\bar{\alpha})$. Set $\bar{\beta}:=\frac{1}{\sqrt{1-l^{2} / 4}} \beta$ which by (57) belongs to the control set $\overline{B_{m}}$. Therefore we conclude that

$$
g_{x_{n}}(\bar{\beta}) \leqslant \frac{1}{\sqrt{1-l^{2} / 4}} g_{x_{n}}(\bar{\alpha}) .
$$


By an easy calculus, the definition of $l$ gives that

$$
\frac{1}{\sqrt{1-l^{2} / 4}} g_{x_{n}}(\bar{\alpha})=-\lambda
$$

which gives a contradiction.

In consequence, we proved that for each integer $n$, there exists $\alpha_{n} \in G_{1}\left(x_{n}\right)$ such that (55) holds. By upper semicontinuity of the multivalued map $x \mapsto \partial W(x)$, it is straightforward to show that

$$
\lim _{n \rightarrow \infty} g_{x_{n}}(\bar{\alpha})=-\lambda \text {; }
$$

which gives the result by (55).

We can apply the well-known Michael's Selection Theorem (see [18] or [5, Theorem 6.5.7, p. 228]) to deduce the existence of a continuous selection $\alpha_{1}(\cdot): \mathcal{D} \rightarrow \overline{B_{m}}$ of $G_{1}$ on the set $\mathcal{D}$. In particular, this means that for every $x \in \mathcal{D}$ we can define the Cauchy problem

$$
\dot{x}(t)=f\left(x(t), \alpha_{1}(x(t))\right), \quad x(0)=x .
$$

The Cauchy-Peano Theorem says that for every $x \in \mathcal{D}$ there exists some solution $x(\cdot)$ of (58) defined on some interval $[0, T]$ and such that $x(t) \in \mathcal{D}$ for every $t \in[0, T]$. (Note that 0 does not belong to the open set $\mathcal{D}$.) The construction of $\alpha_{1}(\cdot)$ yields the following:

Lemma 29. Let $x(\cdot)$ be a solution of (58) defined on some interval $[0, T]$. Then for any $t \in[0, T]$, we have

$$
W(x(t)) \leqslant W(x(0))-\lambda t .
$$

In particular the trajectory can be extended as long as $x(t)$ does not converge to the set $\mathcal{S}$.

Proof. By continuity of $\alpha_{1}(\cdot)$, the function $\theta$ defined by,

$$
\forall t \in[0, T], \quad \theta(t):=W(x(t))
$$

is locally Lipschitz on the interval $[0, T]$. Thus by the classical Chain Rule for limiting subgradients (see [11, Theorem 10.4, p. 62]), for every $t \in(0, T)$ and for every $\eta \in \partial_{L} \theta(t)$, there exists $\zeta \in \partial_{L} W(x(t))$ such that

$$
\eta=\langle\zeta, \dot{x}(t)\rangle=\left\langle\zeta, f\left(x(t), \alpha_{1}(x(t))\right)\right\rangle
$$

Hence by construction of $\alpha_{1}(\cdot)$, we deduce that for every $t \in(0, T), \partial_{L} \theta(t) \leqslant-\lambda$. This means that the function $t \mapsto \theta(t)+\lambda t$ is decreasing, which gives (59). ${ }^{14}$

Actually, (59) says also that the trajectory $x(\cdot)$ remains in the set

$$
S_{W}(W(x(0))):=\left\{x \in \mathbb{R}^{N}: W(x) \leqslant W(x(0))\right\},
$$

which is compact by properness of $W$. In particular this means that as soon as $x(T)$ will belong to $\mathcal{D}$, it will be possible to extend $x(\cdot)$ on some new interval of the form $[0, T+\epsilon]$ (for some $\epsilon>0$ ). This proves Lemma 29.

We conclude that if $x(\cdot)$ is solution of (58) then there exists $T>0$ such that $x(t)$ belongs to $\mathcal{D}$ for any $t \in[0, T)$ and such that $x(T) \in \mathcal{S} ;{ }^{15}$ such a solution will be called a maximal solution of (58). We claim the following result:

\footnotetext{
$\overline{14}$ Here we use the fact that a Lipschitz function $g: \mathbb{R} \rightarrow \mathbb{R}$ is decreasing iff for every $t \in R$ and for every $\zeta \in \partial_{L} g(t)$,

$$
\zeta \leqslant 0
$$
}

15 Notice that since the dynamics $f\left(x, \alpha_{1}(x)\right)$ is bounded on the compact level-set $S_{W}(W(x(0)))$, the $\operatorname{limit}_{t \rightarrow T} x(t)$ exists. 
Lemma 30. Let $x(\cdot)$ be a maximal solution of (58) defined on the interval $[0, T]$ such that $x(T) \neq 0$. Then we have

$$
\Psi(x(T))=-\lambda .
$$

Proof. By semiconcavity of the function $W$ (see Proposition 10) near $x(T) \neq 0$, there exists $\sigma>0$ such that for any $\zeta \in \partial^{P} W(x(T))$, we have

$$
-W(y)+W(x(T))+\sigma\|y-x(T)\|^{2} \geqslant\langle-\zeta, y-x(T)\rangle
$$

whenever $y$ is in a neighborhood of $x(T)$. We deduce that for some $s<T$ and close to $T$, we have

$$
W(x(T))-W(x(s))+\sigma\|x(s)-x(T)\|^{2} \geqslant\langle-\zeta, x(s)-x(T)\rangle .
$$

But Lemma 29 asserts that

$$
W(x(t))-W(x(s)) \leqslant-\lambda(s-t)
$$

whenever $0<s<t<T$. Hence by continuity of $W$ we deduce that

$$
\langle\zeta, x(T)-x(s)\rangle \leqslant W(x(T))-W(x(s))+\sigma\|x(s)-x(T)\|^{2} \leqslant-\lambda(T-s)+\sigma\|x(s)-x(T)\|^{2} .
$$

Now by convexity of the set $f\left(x(T), \overline{B_{m}}\right)$ there exists a sequence $\left(s_{n}\right)_{n}$ and $\alpha_{T}$ in $\overline{B_{m}}$ such that

$$
\lim _{n \rightarrow \infty} \frac{x(T)-x\left(s_{n}\right)}{T-s_{n}}=f\left(x(T), \alpha_{T}\right) .
$$

Consequently, passing to the limit for the sequence $\left(s_{n}\right)_{n}$ in (62), we obtain

$$
\left\langle\zeta, f\left(x(T), \alpha_{T}\right)\right\rangle \leqslant-\lambda .
$$

We can repeat this argument for all $\zeta \in \partial^{P} W(x(T))$, that is,

$$
\forall \zeta \in \partial^{P} W(x(T)), \quad\left\langle\zeta, f\left(x(T), \alpha_{T}\right)\right\rangle \leqslant-\lambda .
$$

Since $\partial^{P} W(x(T))=\partial W(x(T))$ (by semiconcavity of $W$ ), this means that

$$
\Psi(x(T)) \leqslant-\lambda \text {. }
$$

On the other hand, $x(T)$ does not belong to $\mathcal{D}$. By (54) this gives $\Psi(x(T)) \geqslant-\lambda$. Hence we conclude.

In fact, we could now prove that the feedback $\alpha_{1}(\cdot)$ stabilizes the system (1) in the sense of Carathéodory (as we did in [24]). However the feedback $\alpha_{1}(\cdot)$ does maybe not possess the properties that will be needed in the proof of Theorem 5. For example, if $\bar{x}$ is given in $\mathcal{S}$, we do not know what is the set of points $x \in \mathcal{D}$ such that $x(T)=x$ for some $T>0$; this set could have positive measure in $\mathbb{R}^{N}$ ! That is the reason why we are going to detail how we can modify the feedback $\alpha_{1}(\cdot)$ in a neighborhood of $\mathcal{S}$ in such a way that we understand exactly the set of points in $x \in \mathcal{D}$ such that $x(T) \in \mathcal{S}$ for some $T>0$. For that, we denote by $\mathcal{S}_{\lambda}$ the set of $x \in \mathcal{S}$ such that $\Psi(x)=-\lambda$, and we denote by $d_{\mathcal{S}}: \mathbb{R}^{N} \rightarrow$ the distance function to the set $\mathcal{S}$.

Let $\bar{x} \in \mathcal{S} \backslash \mathcal{S}_{\lambda}$. Since $\bar{x}$ belongs to the stratification $\Sigma(W)$, there exist $k \in\{1, \ldots, N\}$ and a unique stratum $S$ of dimension $N-k$ such that $\bar{x} \in S$. Let us first assume that the stratum $S$ is isolated in $\mathcal{S}$, that is such that $S$ does not meet the closure of another stratum of $\Sigma(W)$ meeting $\mathcal{S}$. In particular this means that on every face $F$ of the $k$-simplex $\partial W(\bar{x})$, we have

$$
\Psi(\bar{x})=\max _{\zeta \in F} \min _{\alpha \in \overline{B_{m}}}\langle f(\bar{x}, \alpha), \zeta\rangle \leqslant-\lambda .
$$

Set $C:=\partial W(\bar{x})$ and denote by $F_{1}, \ldots, F_{k+1}$ its $k+1$ faces of dimension $k-1$. Moreover set $K:=f\left(\bar{x}, \overline{B_{m}}\right)$; this set $K$ is compact, strictly convex in the subspace generated by it, and symmetric with respect to the origin. Denote 
by $H_{C}$ the unique subspace of dimension $k$ such that there exists $\zeta \in C$ which satisfies $C \subset \zeta+H_{C}$ and define $E_{C}$ to be the vector subspace of $\mathbb{R}^{N}$ which is spanned by $C$ (note that $E_{C}$ has dimension $k+1$ if $0 \notin C$ and dimension $k$ otherwise). Recall that by semiconcavity of $W$, the normal cone to the stratum $S$ at the point $\bar{x}$ equals

$$
N_{\bar{x}} S=H_{C} .
$$

Define $P_{E_{C}}: \mathbb{R}^{N} \rightarrow E_{C}$ to be the orthogonal projection on $E_{C}$ in $\mathbb{R}^{N}$ and set

$$
\widetilde{K}:=P_{E_{C}}(K) \text {. }
$$

The set $\widetilde{K}$ has the same properties as $K$. Moreover by construction, we note that for every $v \in K$ and for every $\zeta \in C$, we have

$$
\langle v, \zeta\rangle=\left\langle P_{E_{C}}(v)+\left(v-P_{E_{C}}(v)\right), \zeta\right\rangle=\left\langle P_{E_{C}}(v), \zeta\right\rangle
$$

since $v-P_{E_{C}}(v) \perp C$ in $\mathbb{R}^{N}$. Before continuing we recall a notation which will used frequently in the sequel. If $A$ is a subset of $\mathbb{R}^{N}$ then $A^{\perp}$ is defined by

$$
A^{\perp}:=\left\{p \in \mathbb{R}^{N}: q \mapsto\langle q, p\rangle \text { is constant on } A\right\} .
$$

We have the following lemma:

Lemma 31. Assume that $\Psi(\bar{x})<0$. There exists an affine hyperplane $H$ of dimension $k$ in $E_{C}$ which intersects the vector line $C^{\perp}$ at a unique point and such that the convex set $\widetilde{K} \cap H$ has dimension $k$ and satisfies

$$
\forall w \in \partial(\tilde{K} \cap H), \exists \zeta \in C \text { such that }\langle w, \zeta\rangle \leqslant-\lambda .
$$

Proof. Note that the assumption $\Psi(\bar{x})<0$ implies that $0 \notin C$, which gives that $H_{C}$ is a hyperplane in $E_{C}$. Recall that by construction of the set $\widetilde{K}$, we know that

$$
\max _{\zeta \in C} \min _{w \in \widetilde{K}}\langle w, \zeta\rangle=\Psi(\bar{x}) .
$$

Since the stratum $S$ is isolated in $\mathcal{S}$, the concave function $\zeta \mapsto \min _{w \in \widetilde{K}}\langle w, \zeta\rangle$ attains its maximum on $C$ at some $\bar{\zeta}$ which does not belong to a face of $C$. This means that $\bar{\zeta}$ belongs to the relative interior of $C$ in $E_{C}$ and satisfies for every $w \in \widetilde{K}$,

$$
\langle w, \bar{\zeta}\rangle \in[\Psi(\bar{x}),-\Psi(\bar{x})] \subset(-\lambda, \lambda) .
$$

For each face $F_{i}$ of the $k$-simplex $C$, there exists $w_{i} \in \widetilde{K}$ such that

$$
\left\langle w_{i}, \zeta\right\rangle \leqslant-\lambda, \forall \zeta \in F_{i} .
$$

Set $W:=\operatorname{co}\left\{w_{1}, \ldots, w_{k+1}\right\} \subset E_{C}$; we claim that $W$ is a simplex of dimension $k$. We argue by contradiction.

If the dimension of $W$ is strictly less than $k$, then there exists an affine space $L$ of dimension $k-1$ such that $W \subset L$. Since $L^{\perp}$ is a vector plane in $E_{C}$, there exists $u \in H_{C}$ such that the map $w \mapsto\langle w, u\rangle$ is constant on $W$. Since $u \in H_{C}$ and $\bar{\zeta}$ is in the interior of $C$, there exists $t>0$ such that $\bar{\zeta}+t u$ belongs to some face $F_{i}$ of $C$. This gives by construction of $w_{i}$,

$$
\left\langle w_{i}, \bar{\zeta}+t u\right\rangle \leqslant-\lambda
$$

which implies by (65) that $\left\langle w_{i}, u\right\rangle<0$. Finally since $u \in L^{\perp}$ we deduce that

$$
\langle w, u\rangle<0, \quad \forall w \in W .
$$

On the other hand, there exists also $t^{\prime}>0$ such that the vector $\bar{\zeta}-t^{\prime} u$ is on a face $F_{j}$ of $C$. By (65) we get that

$$
\left\langle w_{j}, u\right\rangle>0
$$


which contradicts (66). Hence we proved that $W$ is a $k$-simplex.

Prove now that $W \cap C^{\perp}$ is a singleton. Again we argue by contradiction.

If $W \cap C^{\perp}$ is empty, then this means that there exists $u \in H_{C}$ such that

$$
\langle w, u\rangle>0, \quad \forall w \in W .
$$

As before since $\bar{\zeta}$ belongs to the interior of $C$, there exists $t>0$ such that $\bar{\zeta}$ is on a face of $W$. This means that there exists $i \in\{1, \ldots, k+1\}$ such that $\left\langle w_{i}, \bar{\zeta}+t u\right\rangle \leqslant-\lambda$. By (65) we deduce that $\left\langle w_{i}, u\right\rangle<0$ which contradicts (67). This proves that $W \cap C^{\perp}$ is nonempty. Furthermore, it $W$ meets $C^{\perp}$ in two points, then a face of $W$ meets $C^{\perp}$ which is impossible by construction. As a matter of fact for every face $F$ of the $k$-simplex $W$, there exists an extreme point $\zeta_{F} \in C$ such that $\left\langle w, \zeta_{F}\right\rangle \leqslant-\lambda$ for any $w \in F$. Hence if some $w \in C^{\perp}$ then this would imply that for any $\zeta \in C,\langle w, \zeta\rangle=\left\langle w, \zeta_{F}\right\rangle \leqslant-\lambda$ which is impossible.

Denote by $H$ the unique affine space of dimension $k$ which contains $W$ and prove that it satisfies the required properties. First since $W \subset \widetilde{K}$ is a simplex of dimension $k$, it has dimension $k$. Moreover it intersects $C^{\perp}$ at a unique point. Finally the set of $w \in H$ such that there exists $\zeta \in C$ which satisfies $\langle w, \zeta\rangle \leqslant-\lambda$ is the complement (in $H$ ) of the convex open set

$$
\{w \in H:\langle w, \zeta\rangle>-\lambda, \forall \zeta \in C\} .
$$

But this set contains $W \cap C^{\perp}$ and does not meet the boundary of $W$. This concludes the proof of Lemma 31 .

We can improve this lemma if $\Psi(\bar{x})=0$. In this case, the hyperplane $H$ is not affine; it passes through the origin.

Lemma 32. Assume that $\Psi(\bar{x})=0$. There exists a vector hyperplane $H$ of dimension $k$ in $E_{C}$ which does not contain the vector line $C^{\perp}$ and such that the convex set $\widetilde{K} \cap H$ has dimension $k$ and satisfies

$$
\forall w \in \partial(\widetilde{K} \cap H), \exists \zeta \in C \text { such that }\langle w, \zeta\rangle \leqslant-\lambda .
$$

Proof. We distinguish two cases:

First case: $0 \in C$.

Set $\widehat{K}:=P_{H_{C}}(\widetilde{K})$ where $P_{H_{C}}$ denotes the projection on $H_{C}$. Note that for any $w \in \widetilde{K}$, we have

$$
\langle w, \zeta\rangle=\left\langle P_{H_{C}}(w), \zeta\right\rangle \text {. }
$$

As before since the stratum $S$ is isolated in $\mathcal{S}$, for each face $F_{i}$ there exists $w_{i} \in \widehat{K}$ such that

$$
\left\langle w_{i}, \zeta\right\rangle \leqslant-\lambda, \quad \forall \zeta \in F_{i}
$$

Set $W:=\operatorname{co}\left\{w_{1}, \ldots, w_{k+1}\right\}$; we claim that $W$ is a simplex of dimension $k$. We argue by contradiction.

As before if it is not the case, this means that there exists an affine space $L \subset H_{C}$ of dimension $k-1$ which contains $W$. We deduce that there exists $u \neq 0$ in $L^{\perp}$. Since 0 belongs to the interior of $C$, there is $t>0$ such that $t u$ is on a face $F_{i}$ of $C$. This implies that

$$
\left\langle w_{i}, u\right\rangle \leqslant-\frac{\lambda}{t}<0
$$

which gives that

$$
\langle w, u\rangle<0, \quad \forall w \in W .
$$

But there exists also $t^{\prime}>0$ such that $-t^{\prime} u$ is on a face $F_{j}$. This gives that

$$
\left\langle w_{j}, u\right\rangle>0
$$

which contradicts (69). 
By the same method we can prove that $0 \in W$. Consequently we deduce that $\widehat{K} \cap H_{C}$ is a $k$-simplex which contains the set of $w \in \widehat{K}$ such that

$$
\langle w, \zeta\rangle<-\lambda, \quad \forall \zeta \in C
$$

Hence we conclude that for every $w \in \partial(\widehat{K} \cap H$, there exists $\zeta \in C$ such that $\langle w, \zeta\rangle \leqslant-\lambda$. From definition of $\widehat{K}$ it is clear that there exists an vector hyperplane $H$ of dimension $k$ with $P_{H_{C}}(H)=H_{C}$ such that $\widetilde{K} \cap H$ is a convex set of dimension $k$ and such that (68) is satisfied.

Second case: $0 \notin C$.

In this case there exists $\bar{\zeta} \in C$ such that

$$
\langle w, \bar{\zeta}\rangle=0, \quad \forall w \in \widetilde{K} .
$$

This means that $\widetilde{K}$ is included in the vector hyperplane $H:=(\zeta)^{\perp}$. If we set $\tilde{C}:=P_{H}(C)$ where $P_{H}$ denotes the orthogonal projection on $H$, then we see that for every $\zeta \in C$,

$$
\langle w, \zeta\rangle=\left\langle w, P_{H}(\zeta)\right\rangle, \quad \forall w \in \widetilde{K}
$$

So we can apply the first case. This concludes the proof of Lemma 32.

Lemmas 31 and 32 permits us to show that our control system admits near each point of an isolated stratum of $\mathcal{S}$ a control which makes $W$ and $d_{\mathcal{S}}$ increasing. Let us state the result precisely in the following lemma.

Lemma 33. Let $\bar{x} \in \mathcal{S} \backslash \mathcal{S}_{\lambda}$ and let $S$ be a stratum of dimension $n-k(k \in\{1, \ldots, N\})$ which is isolated in $\mathcal{S}$ and

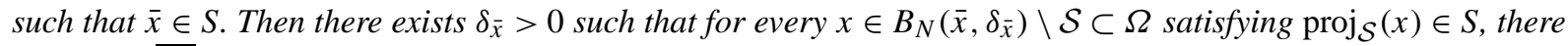
exists $\alpha \in \overline{B_{m}}$ which satisfies

$$
\langle f(x, \alpha), \zeta\rangle \leqslant-\frac{\lambda}{2}, \quad \forall \zeta \in \partial W(x)
$$

and

$$
\langle f(x, \alpha), \xi\rangle \geqslant \delta_{\bar{x}}, \quad \forall \xi \in \partial d_{\mathcal{S}}(x) .
$$

Proof. Since $\bar{S}$ is a smooth manifold with boundary in $\Omega$, the distance function to $\bar{S}$, denoted by $d_{\bar{S}}$ is of class $C^{1}$ on a neighborhood of $\bar{S}$ minus $\bar{S}$ and satisfies for every point $x \notin \bar{S}$ of this neighborhood

$$
\nabla d_{\bar{S}}(x)=\frac{x-p(x)}{\|x-p(x)\|} \in N_{p(x)} \bar{S}
$$

where $p(x)$ is defined by $p(x):=\operatorname{proj}_{\bar{S}}(x)$ (we refer the reader to [12] for the proof of this result). Since $\bar{x} \in S$, there exists some constant $\delta>0$ such that $d_{\bar{S}}$ and $d_{\mathcal{S}}$ coincide on the ball $B_{N}(\bar{x}, \delta)$. In particular, this implies that for every $x \in B_{N}(\bar{x}, \delta) \backslash \mathcal{S}$,

$$
\xi(x):=\nabla d_{\mathcal{S}}(x)=\frac{x-p(x)}{\|x-p(x)\|} \in N_{p(x)} S .
$$

On the other hand, recall that the function $W$ is semiconcave in the ball $B_{N}(\bar{x}, \delta)$. By Proposition 11, this means that there exists some constant $\sigma \geqslant 0$ such that for every pair of points $x, y \in B_{N}(\bar{x}, \delta)$ and for any $\zeta_{x} \in \partial W(x)$, $\zeta_{y} \in \partial W(y)$, we have

$$
\left\langle-\zeta_{y}+\zeta_{x}, y-x\right\rangle \geqslant-\sigma\|y-x\|^{2} .
$$

By (72) we deduce that for any $\zeta \in \partial W(x)$ and for any $\zeta^{\prime} \in \partial W(p(x))$ we have,

$$
\langle\zeta, \xi(x)\rangle \leqslant\left\langle\zeta^{\prime}, \xi(x)\right\rangle+\sigma d_{\mathcal{S}}(x)
$$


Notice that by continuity of $f$ and by smoothness of the manifold $S$, we just need to prove the existence of some positive constant $\delta_{\bar{x}}$ such that (70) and (71) hold whenever $x$ is close to $\bar{x}$ and such that $p(x)=\bar{x}$. As before, we define the elements $C, K, E_{C}, H_{C}$ and $\widetilde{K}$ for the point $\bar{x}$. We distinguish two cases:

First case: $\Psi(\bar{x})<0$.

Let $x \in B_{N}(\bar{x}, \delta)$ such that $x \neq \bar{x}$ and $p(x)=\bar{x}$. The vector $\xi(x) \neq 0$ belongs to $H_{C}$ hence by Lemma 31 there exist $w \in \partial(\widetilde{K} \cap H)$ and $t>0$ such that $P_{H_{C}}(w)=t \xi(x)$, which means that $\langle w, \xi(x)\rangle=t\|\xi(x)\|^{2}=t$. On the other hand, Lemma 31 says also that there is $\zeta^{\prime} \in \partial W(\bar{x})$ such that $\left\langle w, \zeta^{\prime}\right\rangle \leqslant-\lambda$ which gives by (73) for every $\zeta \in \partial W(x)$

$$
\begin{aligned}
\langle w, \zeta\rangle & =\langle t \xi(x), \zeta\rangle+\langle w-t \xi(x), \zeta\rangle \\
& =t\langle\xi(x), \zeta\rangle+\left\langle w-t \xi(x), \zeta^{\prime}\right\rangle \quad\left(\text { since } w-t \xi(x) \perp H_{C}\right) \\
& \leqslant t\left\langle\xi(x), \zeta^{\prime}\right\rangle+t \sigma d_{\mathcal{S}}(x)+\left\langle w-t \xi(x), \zeta^{\prime}\right\rangle \leqslant\left\langle w, \zeta^{\prime}\right\rangle+t \sigma d_{\mathcal{S}}(x) \\
& \leqslant-\lambda+\sigma t d_{\mathcal{S}}(x) .
\end{aligned}
$$

We conclude easily.

Second case: $\Psi(\bar{x})=0$.

Since the vector $\xi(x) \neq 0$ belongs to $H_{C}$, Lemma 32 says that there exist $w \in \partial(\widetilde{K} \cap H)$ and $t>0$ such that $P_{H_{C}}(w)=t \xi(x)$, which means that $\langle w, \xi(x)\rangle=t$. We conclude as before.

The same results holds for strata which are not isolated.

Lemma 34. Assume that $N=2$ and let $\bar{x} \in \mathcal{S} \backslash \mathcal{S}_{\lambda}$. Then there exists $\delta_{\bar{x}}>0$ such that for every $x \in B_{N}\left(\bar{x}, \delta_{\bar{x}} B\right) \backslash$ $\mathcal{S} \subset \Omega$, there exists $\alpha \in \overline{B_{m}}$ which satisfies

$$
\langle f(x, \alpha), \zeta\rangle \leqslant-\frac{\lambda}{2}, \quad \forall \zeta \in \partial W(x)
$$

and

$$
\langle f(x, \alpha), \xi\rangle \geqslant \delta_{\bar{x}}, \quad \forall \xi \in \partial d_{\mathcal{S}}(x) .
$$

Proof. Let us assume that $\bar{x} \in \mathcal{S} \backslash \mathcal{S}_{\lambda} \subset \mathbb{R}^{2}$. Necessarily the point $\bar{x}$ belongs either to $\Sigma^{1}(W)$ either to $\Sigma^{2}(W)$, but since the strata of dimension one are isolated in $\mathcal{S}$, we ever proved the result in that case; hence we can assume that $\bar{x}$ belongs to $\Sigma^{2}(W)$. Moreover notice that by construction of $\lambda$ and $\mathcal{S}$ the point $\bar{x}$ cannot be isolated in $\mathcal{S}$, thus we are in the situation of Fig. 1. This means that in a little ball $\mathcal{B}$ centered at $\bar{x}$, the singular set of $W$ consists in one stratum of dimension 0 , the singleton $\{\bar{x}\}$, plus three strata of dimension one, that is three submanifolds $S_{1}, S_{2}, S_{3}$ of dimension one which join at $\bar{x}$ (we refer the reader to comments concerning Fig. 1 for a complete description of this situation). As before we denote by $\zeta_{1}, \zeta_{2}, \zeta_{3}$ the three extreme points of the convex compact set $C:=\partial W(\bar{x})$ and we denote by $\mathcal{R}_{1}, \mathcal{R}_{2}, \mathcal{R}_{3}$ the three regions where the function $W$ is smooth in $\mathcal{B}$. In addition since the distance function $d_{\mathcal{S}}$ is smooth on a neighborhood of each stratum $S_{i}$, we note that, changing the ball $\mathcal{B}$ if necessary, we can assume that the generalized gradient of $d_{\mathcal{S}}$ writes for every $x \in \mathcal{R}_{1}$ (resp. for every $x \in \mathcal{R}_{2}$ and for every $x \in \mathcal{R}_{3}$ ),

$$
\partial d_{\mathcal{S}}(x)= \begin{cases}\xi_{2}(x) & \text { if } d_{S_{2}}(x)<d_{S_{3}}(x) \\ \xi_{3}(x) & \text { if } d_{S_{3}}(x)<d_{S_{2}}(x) \\ \operatorname{co}\left[\xi_{2}(x), \xi_{3}(x)\right] & \text { if } d_{S_{2}}(x)=d_{S_{3}}(x)\end{cases}
$$

where

$$
\xi_{2}(x):=\frac{x-\operatorname{proj}_{S_{2}}(x)}{\left\|x-\operatorname{proj}_{S_{2}}(x)\right\|} \quad \text { and } \quad \xi_{3}(x):=\frac{x-\operatorname{proj}_{S_{3}}(x)}{\left\|x-\operatorname{proj}_{S_{3}}(x)\right\|}
$$


(respectively with the corresponding formulas for $x$ in the two other regions). Let us now prove our result; since the strata $S_{1}, S_{2}, S_{3}$ are not necessarily included in $\mathcal{S}$, different cases appear:

First case: the three strata $S_{1}, S_{2}, S_{3}$ are in $\mathcal{S}$.

Let us prove that there exists $\delta_{\bar{x}}>0$ such that for every $x \in \mathcal{R}_{1}$ there exists $\alpha \in \overline{B_{m}}$ for which (74) and (75) hold.

By semiconcavity of the function $W$, the upper limit of the sets $\partial d_{\mathcal{S}}(x)$ when $x \in \mathcal{R}_{1}$ tends to $\bar{x}$ is included in the set

$$
\operatorname{co}\left\{\frac{\zeta_{2}-\zeta_{1}}{\left\|\zeta_{2}-\zeta_{1}\right\|}, \frac{\zeta_{3}-\zeta_{1}}{\left\|\zeta_{3}-\zeta_{1}\right\|}\right\}
$$

Therefore it is sufficient to prove that there exists $\alpha \in \overline{B_{m}}$ such that

$$
\left\langle f(\bar{x}, \alpha), \zeta_{1}\right\rangle \leqslant-\lambda
$$

and

$$
\langle f(\bar{x}, \alpha), \xi\rangle>0, \quad \forall \xi \in\left[\zeta_{2}-\zeta_{1}, \zeta_{3}-\zeta_{1}\right] .
$$

But since the strata $S_{2}$ and $S_{3}$ belong to $\mathcal{S}$, every control $\alpha \in \overline{B_{m}}$ such that $\left\langle f(x \alpha), \zeta_{1}\right\rangle \leqslant-\lambda$ satisfies

$$
\left\langle f(x, \alpha), \zeta_{i}\right\rangle>-\lambda, \quad \forall i=1,2,
$$

which implies

$$
\left\langle f(x, \alpha), \xi_{i}\right\rangle>0, \quad \forall \xi \in\left[\zeta_{2}-\zeta_{1}, \zeta_{3}-\zeta_{1}\right]
$$

In conclusion we get that there exists $\alpha \in \overline{B_{m}}$ which satisfies (76) and (77). The same conclusion hold for the regions $\mathcal{R}_{2}$ and $\mathcal{R}_{3}$; we conclude easily.

Second case: one of the $S_{i}$ 's is not in $\mathcal{S}$.

Without loss of generality we can assume that $S_{1}$ does not meet $\mathcal{S}$. If $x \in \mathcal{B}$ is in the region $\mathcal{R}_{1}$ then the first case gives the result. Otherwise setting $\mathcal{R}=\mathcal{R}_{2} \cup \mathcal{R}_{3} \cup S_{1}$, we note that the upper limit of the sets $\partial W(x)$ when $x \in \mathcal{R}$ tends to $\bar{x}$ is included in the segment $\left[\zeta_{2}, \zeta_{3}\right]$ and that the upper limit of the sets $\partial d_{\mathcal{S}}(x)$ when $x \in \mathcal{R}$ tends to $\bar{x}$ is included in the set

$$
\operatorname{co}\left\{\frac{\zeta_{1}-\zeta_{2}}{\left\|\zeta_{1}-\zeta_{2}\right\|}, \frac{\zeta_{1}-\zeta_{3}}{\left\|\zeta_{1}-\zeta_{3}\right\|}\right\}
$$

Therefore it is sufficient to prove that there exists $\alpha \in \overline{B_{m}}$ such that

$$
\langle f(\bar{x}, \alpha), \zeta\rangle \leqslant-\lambda, \quad \forall \zeta \in\left[\zeta_{2}, \zeta_{3}\right]
$$

and

$$
\left\langle f(\bar{x}, \xi\rangle>0, \quad \forall \xi \in\left[\zeta_{1}-\zeta_{2}, \zeta_{1}-\zeta_{3}\right] .\right.
$$

But since the stratum $S_{1}$ is not in $\mathcal{S}$, there exists $\alpha \in \overline{B_{m}}$ such that

$$
\langle f(\bar{x}, \alpha), \zeta\rangle \leqslant-\lambda, \quad \forall \zeta \in\left[\zeta_{2}, \zeta_{3}\right] .
$$

In consequence since both strata $S_{2}$ and $S_{3}$ are in $\mathcal{S}$ we deduce that for such $\alpha$,

$$
\langle f(x, \alpha), \xi\rangle>0, \quad \forall \xi \in\left[\zeta_{1}-\zeta_{2}, \zeta_{1}-\zeta_{3}\right] .
$$

This conclude the proof of the second case.

Third case: two of the $S_{i}$ 's are not in $\mathcal{S}$.

Without loss of generality we can assume that $S_{2}$ and $S_{3}$ do not meet $\mathcal{S}$. Notice that since $S_{2}$ (resp. $S_{3}$ ) does not meet the set $\mathcal{S}$, there exists some control $\alpha_{2} \in \overline{B_{m}}$ (resp. $\alpha_{3} \in \overline{B_{m}}$ ) such that

$$
\left\langle f\left(\bar{x}, \alpha_{2}\right), \zeta\right\rangle \leqslant-\lambda, \quad \forall \zeta \in\left[\zeta_{1}, \zeta_{2}\right]
$$


(Respectively

$$
\left.\left\langle f\left(\bar{x}, \alpha_{3}\right), \zeta\right\rangle \leqslant-\lambda, \quad \forall \zeta \in\left[\zeta_{1}, \zeta_{3}\right] .\right)
$$

On the other hand, since the stratum $S_{1}$ meets $\mathcal{S}$, we have

$$
\left\langle f\left(\bar{x}, \alpha_{2}\right), \zeta_{3}-\zeta_{2}\right\rangle>0 \text {. }
$$

(Respectively

$$
\left.\left\langle f\left(\bar{x}, \alpha_{3}\right), \zeta_{2}-\zeta_{3}\right\rangle>0 .\right)
$$

This implies easily that Lemma 34 holds in the third case.

In order to conclude the construction of our feedback, we need to define the concept of bifurcation point of a closed-loop system.

Definition 35. Assume that we are given a closed subset $\mathcal{F}$ of $\Omega$, an open set $\mathcal{U} \subset \Omega$ containing $\mathcal{F}$ and a feedback $\alpha(\cdot): \mathcal{U} \rightarrow \overline{B_{m}}$ which is continuous on $\mathcal{U} \backslash \mathcal{F}$ and such that the corresponding closed-loop system

$$
\dot{x}=f(x, \alpha(x)),
$$

has always local Carathéodory solutions in $\mathcal{U}$ (that is for any initial state in $\mathcal{U}$ ). Then a point $x$ in $\mathcal{F}$ is called a bifurcation point of the feedback $\alpha(\cdot)$ if there exist $t>0$ and a Carathéodory trajectory $x(\cdot)$ of $(80)$ defined on $[0, t]$ such that

$$
x(t)=x .
$$

Using a locally finite covering of the set $\mathcal{S} \backslash \mathcal{S}_{\lambda}$ by the balls given by Lemma 34, we deduce that we can construct in a neighborhood of $\mathcal{S}$ in $\Omega$ a feedback which makes $W$ decreasing and with nice properties of bifurcation (see lemma below and Figs. 2, 3). Furthermore, we note that the additional assumption we made on the smoothness of the vector fields $f_{1}, \ldots, f_{m}$ is in fact not necessary. Actually, the smoothness of the $f_{i}$ 's helped us, by Lemma 26, to truncate easily and properly the bifurcation strata of the singular set $\Sigma(W)$ into Lipschitz manifolds with boundary, and then to get a nice set $\mathcal{S}$. If the vector fields are only assumed to be locally Lipschitz, this truncation can still be done but in a different way. In any case, based on Lemma 34 and constructing meticulously a feedback step by step in a neighborhood of $\mathcal{S}$, we can prove the following:

Lemma 36. Assume that $N=2$. There exists some open neighborhood $\mathcal{V}$ of the set $\mathcal{S}$, a closed subset $\widetilde{\mathcal{S}}$ of $\mathcal{S}$ and a feedback $\alpha_{2}(\cdot): \mathcal{V} \rightarrow \mathbb{R}^{m}$ such that the following properties are satisfied:

(i) the set $\widetilde{\mathcal{S}}$ is closed in $\Omega$ and stratified by strata of dimension less than $N-1$;

(ii) the feedback $\alpha_{2}(\cdot)$ is smooth on $\mathcal{V} \backslash \widetilde{\mathcal{S}}$;

(iii) for every $x \in \mathcal{V} \backslash \widetilde{\mathcal{S}}$, we have

$$
\left\langle f\left(x, \alpha_{2}(x)\right), \zeta\right\rangle \leqslant-\frac{\lambda}{2}, \quad \forall \zeta \in \partial W(x)
$$

(iv) for every $x \in \mathcal{V}$, the Carathéodory solutions of $\dot{x}=f\left(x, \alpha_{2}(x)\right), x(0)=x$ exist locally and satisfy

$$
W(x(t)) \leqslant W(x(0))-\frac{\lambda}{2} t,
$$

for any $t \geqslant 0$ such that $x(t) \in \mathcal{V}$;

(v) for each stratum $S$ of $\widetilde{S}$ of dimension $k\left(k \in\{1, \ldots, N-1)\right.$, the set of bifurcation points of $\alpha_{2}(\cdot)$ in $S$ is a finite union of smooth connected submanifolds of dimension strictly less than $k$ (whenever it is nonempty); 
(vi) if we denote by $\widetilde{\mathcal{S}}_{b}$ the set of bifurcation points of $\alpha_{2}(\cdot)$ in $\widetilde{\mathcal{S}}$, then for every $x \in \widetilde{\mathcal{S}}_{b}$ there exists $\delta>0$ such that $x+\delta B \subset \mathcal{V}$ and such that for every $y \in \widetilde{\mathcal{S}}_{b} \cap(x+\delta B)$, the Cauchy problem $\dot{x}=-f\left(x, \alpha_{2}(x)\right), x(0)=y$ admits locally a unique solution which can in addition be defined on the interval $[0, \delta)$; moreover the flow of this dynamical system is continuous on $\widetilde{\mathcal{S}}_{b} \cap(x+\delta B) \times[0, \delta) .{ }^{16}$
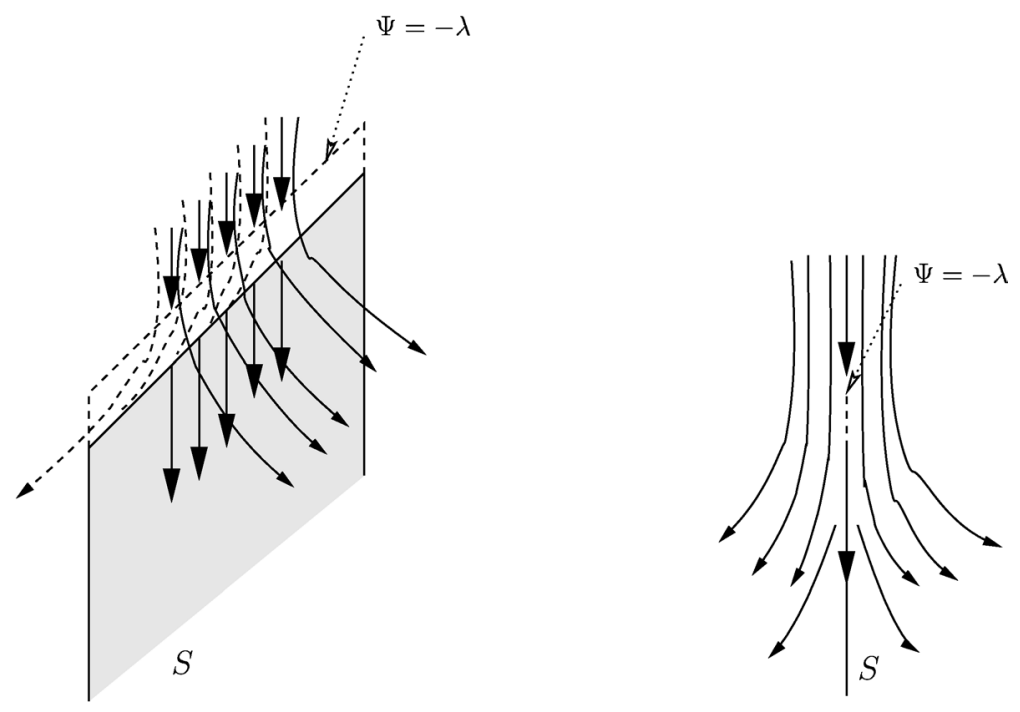

Fig. 2. Bifurcation of the feedback near an isolated stratum.
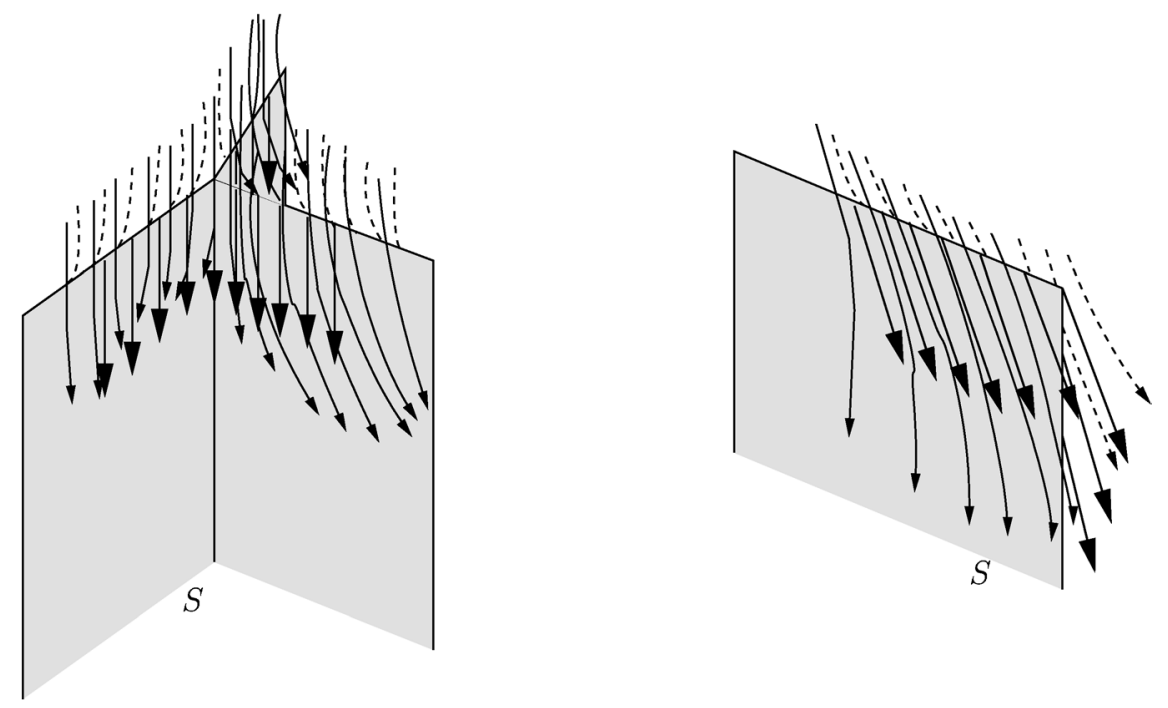

Fig. 3. Bifurcation of the feedback in the general case.

\footnotetext{
16 We call flow of the dynamical system $\dot{x}=-f\left(x, \alpha_{2}(x)\right)$ on $\widetilde{\mathcal{S}}_{b} \cap(x+\delta B) \times[0, \delta)$, the function $\theta: \widetilde{\mathcal{S}}_{b} \cap(x+\delta B) \times[0, \delta) \rightarrow \mathcal{V}$ defined by $\theta(y, t):=x(t)$ where $x(\cdot)$ is the unique solution of $\dot{x}=-f\left(x, \alpha_{2}(x)\right), x(0)=y$.
} 
In order to obtain our stabilizing feedback $\alpha(\cdot)$, we now paste $\alpha_{2}(\cdot)$ to our first stabilizing feedback $\alpha_{1}(\cdot)$. Let $\mathcal{W}$ be some open neighborhood of $\widetilde{\mathcal{S}}$ such that $\overline{\mathcal{W}} \subset \mathcal{V}$; we proceed as follows:

Set $\widetilde{D}:=\Omega \backslash \widetilde{\mathcal{S}}$ and define the new multifunction $G: \widetilde{\mathcal{D}} \rightarrow \overline{B_{m}}$ by

$$
G(x):= \begin{cases}\alpha_{1}(x) & \text { if } x \notin \mathcal{V}, \\ \left\{\alpha \in \overline{B_{m}}: \forall \zeta \in \partial W(x),\langle f(x, \alpha), \zeta\rangle \leqslant-\lambda / 2\right\} & \text { if } x \in \mathcal{V} \backslash \overline{\mathcal{W}}, \\ \alpha_{2}(x) & \text { if } x \in \overline{\mathcal{W}} .\end{cases}
$$

Since $\alpha_{1}(\cdot)$ and $\alpha_{2}(\cdot)$ are continuous and since $G$ is lower semicontinuous on $\mathcal{V} \backslash \overline{\mathcal{W}}$ (by Lemma 28 applied with $\lambda=\lambda / 2)$, it is clear that the multifunction $G$ is lower semicontinuous on $\widetilde{\mathcal{D}}$. Hence by Michael's Selection Theorem it admits a continuous selection $\alpha(\cdot)$ on $\widetilde{\mathcal{D}}$ which satisfies by Lemma 36 (iii) and by construction of $G$ and $\alpha_{1}(\cdot)$,

$$
\forall x \in \widetilde{\mathcal{D}}, \forall \zeta \in \partial W(x), \quad\left\langle f\left(x, \alpha_{2}(x)\right), \zeta\right\rangle \leqslant-\frac{\lambda}{2} .
$$

Therefore up to regularization the feedback $\alpha(\cdot)$ by convolution on $\mathcal{V} \backslash \overline{\mathcal{W}}$ if necessary, we can assume that $\alpha(\cdot)$ is smooth on $\widetilde{\mathcal{D}}$ and satisfies

$$
\forall x \in \widetilde{\mathcal{D}}, \forall \zeta \in \partial W(x), \quad\left\langle f\left(x, \alpha_{2}(x)\right), \zeta\right\rangle \leqslant-\frac{\lambda}{4} .
$$

Consequently by Lemma 36(iv) and by Lemma 29 (applied with $\lambda=\lambda / 4$ ), the Carathéodory trajectories $x(\cdot$ ) of the closed-loop system $\dot{x}=f(x, \alpha(x))$ satisfy for any $t \geqslant 0$,

$$
W(x(t))-W(x(0)) \leqslant-\frac{\lambda}{4} t .
$$

From properties of $W$, this proves easily that the feedback $\alpha(\cdot)$ is stabilizing in the sense of Carathéodory. Furthermore by construction, the bifurcation points of $\alpha(\cdot)$ correspond exactly to the bifurcation points of $\alpha_{2}(\cdot)$. In other terms, we have proved the following result which will be fundamental for the proof of Theorem 5 .

Theorem 37. Assume that $N=2$. If the system (1) is GAC then there exists a feedback $\alpha(\cdot): \mathbb{R}^{N} \rightarrow \overline{B_{m}}$ and a set $\mathcal{S} \subset \mathbb{R}^{N} \backslash\{0\}$ which satisfy the following properties:

(i) the set $\mathcal{S}$ is closed in $\Omega$ and stratified by strata of dimension less than $N-1$;

(ii) the feedback $\alpha(\cdot)$ is smooth in $\Omega \backslash \mathcal{S}$;

(iii) the closed-loop system $\dot{x}=f(x, \alpha(x))$ is GAS in the sense of Carathéodory;

(iv) for every stratum $S$ of $\mathcal{S}$ of dimension $k$ (with $k \in\{1, \ldots, N-1\}$ ), the set of bifurcations points of $\alpha(\cdot)$ in $S$ is a finite union of smooth connected submanifolds of dimension strictly less than $k$ (whenever it is nonempty);

(v) if we denote by $\mathcal{S}_{b}$ the set of bifurcation points of $\alpha(\cdot)$ in $\mathcal{S}$, then for every $x \in \mathcal{S}_{b}$ the Cauchy problem $\dot{x}=-f\left(x, \alpha_{2}(x)\right), x(0)=x$ admits locally a unique solution which can in addition be defined on the interval $[0, \infty)$; moreover the flow of this dynamical system is continuous on $\mathcal{S}_{b} \times[0, \infty)$.

We are now ready to prove the main result of the paper.

\subsection{Proof of Theorem 5}

As we said, Theorem 5 is a direct consequence of Theorem 37. Here $N=2$, denote by $\theta$ the flow of the system $\dot{x}=-f(x, \alpha(x))$ on $\mathcal{S}_{b} \times[0, \infty)$. For each stratum $S$ of the set $\mathcal{S}$, we consider $\widetilde{S}_{b}$ defined by

$$
\widetilde{S}_{b}:=\left\{\theta(x, t): x \in S \cap \mathcal{S}_{b}, t \geqslant 0\right\} .
$$

By (iv) and (v) of Theorem 37, this set is a submanifold of $\mathbb{R}^{N}$ of dimension less than $N-1$. Thus if we consider $\widetilde{\mathcal{S}}$ to be the union of all the $\widetilde{S}$, it has measure zero (since the number of strata of $\mathcal{S}$ is at most countable). And 
moreover by (iii) every $x \in \mathbb{R}^{N} \backslash \widetilde{\mathcal{S}}$ is stabilized to the origin without meeting the set $\mathcal{S}$. To conclude the proof of Theorem 5, we need the following lemma.

Lemma 38. There exists some function $\rho: \mathbb{R}^{N} \rightarrow[0, \infty)$ which is of class $C^{1}$ on $\mathbb{R}^{N} \backslash\{0\}$, continuous at the origin and such that

(i) $\rho(0)=0$;

(ii) $\rho(x)=0 \Leftrightarrow x \in \mathcal{S}$;

(iii) $\nabla \rho(x)=0, \forall x \in \mathcal{S}$.

Proof. The closure of each stratum $S$ of the set $\mathcal{S}$ is a smooth submanifold with boundary. Hence the function

$$
x \mapsto d_{S}(x)^{2}
$$

is of class $C^{1}$ on a neighborhood of $\bar{S}$ and satisfies

$$
\nabla\left[d_{S}^{2}\right](x)=0, \quad \forall x \in \bar{S} .
$$

This means that for each stratum $S$ of the set $\mathcal{S}$, there exists a function $h_{S}: \mathbb{R}^{N} \rightarrow[0,1]$ of class $C^{1}$ which satisfies

$$
\left\{\begin{array}{l}
h_{S}(x)=0 \Longleftrightarrow x \in \bar{S} \\
\nabla h_{S}(x)=0, \quad \forall x \in \bar{S} .
\end{array}\right.
$$

Since the set $\mathcal{S}$ is a locally finite union of such strata in $\Omega=\mathbb{R}^{N} \backslash\{0\}$, this means that on each open subset $\mathcal{O} \subset \Omega$ which is relatively compact in $\Omega$ (i.e. such that $\overline{\mathcal{O}} \subset \Omega$ ), the set $\mathcal{S}$ is included in finite union of strata $S_{1}, \ldots, S_{p}$. Define on $\mathcal{O}$ the function $k_{\mathcal{O}}$ by

$$
\forall x \in \mathcal{O}, \quad k_{\mathcal{O}}(x):=\|x\| \prod_{i=1}^{p} h_{S_{i}}(x) \quad(\leqslant\|x\|) .
$$

It is straightforward to show that the function $k_{\mathcal{O}}$ satisfies the assertions (ii) and (iii) on $\mathcal{O}$. Consequently, we conclude by pasting together the different functions $h_{\mathcal{O}}$ by a smooth partition of unity subordinate to a locally finite (and relatively compact) covering of $\Omega$.

Now set for every $x \in \mathbb{R}^{N}$,

$$
\tilde{\alpha}(x):=\rho(x) \alpha(x) .
$$

The feedback $\tilde{\alpha}(\cdot)$ is clearly continuous on $\mathbb{R}^{N}$ and of class $C^{1}$ where $\alpha(\cdot)$ is smooth, that is outside $\{0\} \cup \mathcal{S}$. Besides since $\nabla \rho=0$ on $\mathcal{S}$, we get that $\tilde{\alpha}(\cdot)$ is of class $C^{1}$ on the set $\mathcal{S}$. Furthermore since the scalar function has positive values outside $\{0\} \cup \mathcal{S}$, it is straightforward to show that the closed-loop system

$$
\dot{x}=f(x, \tilde{\alpha}(x))
$$

is AGAS, which completes the proof of Theorem 5.

\section{Additional comments on the two preceding sections}

\subsection{Control systems with drift}

We could wonder if Theorem 5 holds in the general case of control system with drift, of the form

$$
\dot{x}=f(x, \alpha):=f_{0}(x)+\sum_{i=1}^{m} \alpha_{i} f_{i}(x),
$$


where $f_{0}, f_{1}, \ldots, f_{m}$ are locally Lipschitz vector fields on $\mathbb{R}^{N}$ and where the control $\alpha$ is in $\overline{B_{m}}$. The answer is no! This gives us the opportunity to draw attention to the importance of the Lyapunov stability in the conclusions of Theorem 5. The absence of drift permitted us to vanish the dynamics at the points of discontinuity of the repulsive stabilizing feedback and hence to avoid the escape of the state far from the equilibrium. However in the case of a control system with drift, we cannot vanish the dynamics and then we cannot insure the Lyapunov stability of the smooth AGAS feedback. In fact, one sees easily that Theorem 37 remains in the case of GAC control systems with drift. Hence it is natural to ask the following:

Open Question. If the system (81) is GAC, does there exist a smooth (outside the origin) feedback $\alpha(\cdot): \mathbb{R}^{n} \rightarrow \mathbb{R}^{m}$ such that for almost every $x \in \mathbb{R}^{N}$, the solution of the closed-loop system

$$
\dot{x}(t)=f_{0}(x(t))+\sum_{i=1}^{m} \alpha_{i}(x(t)) f_{i}(x(t)), \quad x(0)=x
$$

converge to the origin as $t$ tends to infinity?

\subsection{Control systems on manifolds}

All the results that we develop in the present paper remain valid on smooth manifolds. If we want to prove Theorems 3 on a smooth manifold, we have to apply the same procedure of regularization as the one given in Section 1.4. Let be given a semiconcave control-Lyapunov function for the system (1) on a smooth manifold $M$ (of course this means that (1) is defined by vector fields defined on a smooth manifold $M$ ), starting from a open cube included in some chart, we regularize step by step the CLF everywhere. From Theorem 3, we can prove the existence of AGAS feedbacks by the construction given in Sections 2.1 and 2.2. Since we do not want to write these results and hence to have to introduce the natural notions of viscosity solutions and semiconcavity on smooth manifolds, we prefer stopping our remark here. We notice that if the reader just wants to develop our results on a smooth submanifold of the Euclidean space (which is indeed sufficient by Whitney's embedding theorem), he can read the paper of Sontag [32] which explains how to extend a given system to the ambient space and then to use the known results in the case of the Euclidean space.

\subsection{Invariance, repulsivity and last comments}

In Theorem 37, we constructed a stabilizing feedback $\alpha(\cdot)$ (in the sense of Carathéodory) which is smooth outside a certain closed set $\mathcal{S}$ (closed in $\mathbb{R}^{N} \backslash\{0\}$ ). As we saw, the set $\mathbb{R}^{N} \backslash \mathcal{S}$ is not necessarily invariant with respect to the closed-loop system given by $\alpha(\cdot)$; some bifurcation singularities can appear along the trajectories of the system. We can wonder if it is possible to construct a closed set $\mathcal{S}$ in $\mathbb{R}^{N} \backslash\{0\}$ and a stabilizing feedback $\alpha(\cdot)$ such that the set $\mathbb{R}^{N} \backslash \mathcal{S}$ is invariant under the dynamics $\dot{x}=f(x, \alpha(x))$. The answer is probably no; Theorem 37 allows us to construct such an invariant couple $(\mathcal{S}, \alpha)$ but the set $\mathcal{S}$ has no reason to be closed. Let us state the following definition (see [4]):

Definition 39. The closed-loop system (2) is said to be almost (smoothly) exponentially stable in the region $\mathcal{R} \subset \mathbb{R}^{N}$ if the feedback $\alpha(\cdot)$ is smooth ${ }^{17}$ on $\mathcal{R}$ and if the following holds:

(i) for all initial state $x_{0} \in \mathcal{R}$ the closed-loop system (2) admits a unique solution and this solution remains in $\mathcal{R}$ for all $t \geqslant 0$;

\footnotetext{
17 We have to make clear what we mean by smooth if the set $\mathcal{R}$ is not open. We say that $\alpha(\cdot): \mathcal{R} \rightarrow \overline{B_{m}}$ is smooth if there exists an open set $\mathcal{D}$ which contains $\mathcal{R}$ and a smooth function $\alpha^{\prime}(\cdot): \mathcal{D} \rightarrow \overline{B_{m}}$ such that $\alpha^{\prime}(\cdot)=\alpha(\cdot)$ on $\mathcal{R}$.
} 
(ii) there exists a continuous positive definite function $\Psi: \mathbb{R}^{N} \rightarrow \mathbb{R}$ such that for all $t \geqslant 0$ one has, along the trajectories of the closed-loop system,

$$
\Psi(x(t)) \leqslant \Psi\left(x_{0}\right) \mathrm{e}^{-t} .
$$

As we said above, Theorem 37 leads naturally to the following:

Theorem 40. If the system (1) is GAC then there exists a dense subset $\mathcal{R} \subset \mathbb{R}^{N}$ of full measure and a feedback $\alpha(\cdot): \Omega \rightarrow \overline{B_{m}}$ such that (the closed-loop system 2 ) is almost exponentially stable.

Proof. According to the proof of Theorem 5, we set $\mathcal{R}:=\mathbb{R}^{N} \backslash \mathcal{S}$ and the result follows.

As we said above, the region $\mathcal{R}$ that we construct may not be open. Hence one question arises:

Open Question. Under what condition on the GAC control system (1) does there exist an open dense set $\mathcal{D}$ of $\mathbb{R}^{N} \backslash\{0\}$ associated with a smooth feedback $\alpha_{\mathcal{D}}(\cdot): \mathcal{D} \rightarrow \mathbb{R}^{m}$ such that the closed-loop system $\dot{x}=f\left(x, \alpha_{\mathcal{D}}\right)$ is GAS at the origin and such that $\mathcal{D}$ is invariant with respect to this system?

In [24], we saw that some control systems possess a stronger property of stabilizability. There are systems which admit a stabilizing feedback in the sense of Carathéodory which is smooth on an open dense set of full measure $\mathcal{D} \subset \mathbb{R}^{N} \backslash\{0\}$, and such that every Carathéodory solution of the closed-loop system evolves in $\mathcal{D}$ for any positive time (this property is stronger than the invariance of $\mathcal{D}$ ). We say that such control systems possess a smooth repulsive stabilizing (SRS) feedback. In [24], we showed some control systems which cannot have SRS feedbacks and we gave some sufficient conditions on the CLF of a control system which imply the existence of SRS feedbacks. The problem of the existence of SRS feedbacks seems quite difficult; we refer the reader to [27,28] for first results on this problem.

\section{Acknowledgements}

The author is very indebted to the referee who read the paper with great care, pointed out a large number of misprints and detected a serious error in the first version of the paper.

\section{Appendix A}

Here we present an important corollary of a result concerning semiconcavity, which was proven by Alberti, Ambrosio and Cannarsa in [2] (the reader is also referred to the recent book on semiconcavity written recently by Cannarsa and Sinestrari, see [8]). Given a semiconcave function

$$
u: \Omega \rightarrow \mathbb{R}
$$

they established a link between the Boulingand's contingent cone to the singular set $\Sigma(u)$ at $x \in \Omega$, and the generalized gradient $\partial u(x)$. Below, we prove this result in the very particular case of semiconcave function with nice singular set.

Assume that there exists $k \in\{1, \ldots, N\}$ and a set $S \subset \Omega$ which satisfy the following properties:

(a) $S$ is a smooth submanifold (without boundary) of dimension $N-k$ in $\Omega$, 
(b) there exists $k+1$ maps $\zeta_{1}(\cdot), \ldots, \zeta_{k+1}(\cdot)$ which are smooth on $S$ and such that for every $x \in S$,

$$
\partial u(x)=\operatorname{co}\left\{\zeta_{1}(x), \ldots, \zeta_{k+1}(x)\right\}
$$

(c) for every $x \in S$, the convex set $\partial u(x)$ has dimension $k$.

If $A$ is a given set in $\mathbb{R}^{N}$, we denote by $A^{\perp}$ the set defined by

$$
A^{\perp}:=\left\{p \in \mathbb{R}^{N}: q \mapsto\langle q, p\rangle \text { is constant on } A\right\} .
$$

The following result follows.

Proposition 41. For every $x \in S$,

$$
T_{x} S=\partial u(x)^{\perp} .
$$

Proof. Fix $p \in T_{x} S$ and prove that $p \in \partial u(x)^{\perp}$. Let $\zeta_{1}, \zeta_{2} \in \partial u(x)$; we have to prove that $\left\langle\zeta_{1}, p\right\rangle=\left\langle\zeta_{2}, p\right\rangle$.

First, note that without loss of generality we can assume that $\|p\|=1$. By definition of the tangent space, there exists a sequence $\left(x_{n}\right)_{n}$ in $S$ such that

$$
\lim _{n \rightarrow \infty} \frac{x_{n}-x}{\left\|x_{n}-x\right\|}=p
$$

Moreover by (b), there exists some sequence $\left(q_{n}\right)_{n}$ which converges to $\zeta_{2}$ in $\mathbb{R}^{N}$. In addition by Proposition 10 , there exists $\sigma>0$ and some neighborhood $\mathcal{V}$ of $x$ such that

$$
-u(z)+u(y)+\sigma\|z-y\|^{2} \geqslant\langle-\zeta, z-y\rangle,
$$

for all $y, z \in \mathcal{V}$ and $\zeta \in \partial u(y)$. Applying this inequality with $y=x, z=x_{n}$ and $\zeta=\zeta_{1}$ gives for each $n$,

$$
-u\left(x_{n}\right)+u(x)+\sigma\left\|x_{n}-x\right\|^{2} \geqslant\left\langle-\zeta_{1}, x_{n}-x\right\rangle
$$

Now applying the same inequality with $y=x_{n}, z=x$ and $\zeta=q_{n}$ gives for each $n$,

$$
-u(x)+u\left(x_{n}\right)+\sigma\left\|x-x_{n}\right\|^{2} \geqslant\left\langle-q_{n}, x-x_{n}\right\rangle .
$$

Summing both inequalities and letting $n \rightarrow \infty$ implies

$$
\left\langle\zeta_{2}, p\right\rangle \leqslant\left\langle\zeta_{1}, p\right\rangle \text {. }
$$

Inverting the roles played by $\zeta_{1}$ and $\zeta_{2}$, we conclude.

Prove now that $\partial u(x)^{\perp} \subset T_{x} S$. By (c), $\partial u(x)^{\perp}$ is a vector subspace of dimension $N-k$, hence it coincides with $T_{x} S$. The proof of Proposition 41 is complete.

\section{References}

[1] P. Albano, P. Cannarsa, Structural properties of singularities of semiconcave functions, Ann. Scuola Norm. Sup. Pisa Cl. Sci. (4) 28 (4) (1999) 719-740.

[2] G. Alberti, L. Ambrosio, P. Cannarsa, On the singularities of convex functions, Manuscripta Math. 76 (3-4) (1992) $421-435$.

[3] F. Ancona, A. Bressan, Patchy vector fields and asymptotic stabilization, ESAIM Control Optim. Calc. Var. 4 (1999) $445-471$.

[4] A. Astolfi, Discontinuous control of nonholonomic systems, Systems Control Lett. 27 (1) (1996) 37-45.

[5] J.-P. Aubin, Viability Theory, Birkhäuser Boston, Boston MA, 1991.

[6] M. Bardi, I. Capuzzo-Dolcetta, Optimal Control and Viscosity Solutions of Hamilton-Jacobi-Bellman Equations, Birkhäuser Boston, Boston, MA, 1997.

[7] R.W. Brockett, Asymptotic stability and feedback stabilization, in: R.W. Brockett, R.S. Millman, H.J. Sussmann (Eds.), Differential Geometric Control Theory, Birkhäuser, Boston, 1983, pp. 181-191. 
[8] P. Cannarsa, C. Sinestrari, Semiconcave Functions, Hamilton-Jacobi Equations, and Optimal Control, Progress in Nonlinear Differential Equations and Their Applications, vol. 58, Birkhäuser Boston, Boston, MA, 2004.

[9] F.H. Clarke, Optimization and Nonsmooth Analysis, Wiley-Interscience, New York, 1983; Republished: Classics Appl. Math., vol. 5, SIAM, 1990.

[10] F.H. Clarke, Yu.S. Ledyaev, E.D. Sontag, A.I. Subbotin, Asymptotic controllability implies feedback stabilization, IEEE Trans. Automat. Control 42 (1997) 1394-1407.

[11] F.H. Clarke, Yu.S. Ledyaev, R.J. Stern, P.R. Wolenski, Nonsmooth Analysis and Control Theory, Graduate Texts in Math., vol. 178, Springer-Verlag, New York, 1998.

[12] F.H. Clarke, R.J. Stern, P.R. Wolenski, Proximal smoothness and the lower- $C^{2}$ property, J. Convex Anal. 2 (1995) $117-145$.

[13] J.-M. Coron, On the stabilization of some nonlinear control systems: results, tools, and applications, in: Nonlinear Analysis, Differential Equations and Control (Montreal, QC, 1998), Kluwer Academic, Dordrecht, 1999, pp. 307-367.

[14] M.G. Crandall, P.-L. Lions, Viscosity solutions of Hamilton-Jacobi equations, Trans. Amer. Math. Soc. 277 (1) (1983) 1-42.

[15] F.R. Gantmacher, The Theory of Matrices. Vol. 1, AMS Chelsea Publishing, Providence, RI, 1998.

[16] M. Goresky, R. MacPherson, Stratified Morse Theory, Springer-Verlag, Berlin, 1988.

[17] P.-L. Lions, Generalized Solutions of Hamilton-Jacobi Equations, Pitman, Boston, MA, 1982 (Advanced Publishing Program).

[18] E. Michael, Continuous selections. I, Ann. of Math. (2) 63 (1956) 361-382.

[19] A. Rantzer, A dual to Lyapunov stability theorem, Systems Control Lett. 42 (3) (2000) 161-168.

[20] A. Rantzer, A converse Lyapunov stability theorem, Personnal communication.

[21] L. Rifford, Problèmes de stabilisation en théorie du controle, PhD thesis, Université Claude Bernard Lyon I, 2000.

[22] L. Rifford, Stabilisation des systèmes globalement asymptotiquement commandables, C. R. Acad. Sci. Paris Sér. I Math. 330 (3) (2000) 211-216.

[23] L. Rifford, Existence of Lipschitz and semiconcave control-Lyapunov functions, SIAM J. Control Optim. 39 (4) (2000) $1043-1064$.

[24] L. Rifford, Semiconcave control-Lyapunov functions and stabilizing feedbacks, SIAM J. Control Optim. 41 (3) (2002) 659-681.

[25] L. Rifford, Singularities of viscosity solutions and the stabilization problem in the plane, Indiana Univ. Math. J. 52 (5) (2003) $1373-1396$.

[26] L. Rifford, A Morse-Sard theorem for the distance function on Riemannian manifolds, Manuscripta Math. 113 (2004) $251-265$.

[27] L. Rifford, On the existence of local smooth repulsive stabilizing feedbacks in dimension three, in preparation.

[28] L. Rifford, The stabilization problem on surfaces, Rend. Semin. Mat. Torino, submitted for publication.

[29] R.T. Rockafellar, Convex Analysis, Princeton University Press, Princeton, NJ, 1997. Reprint of the 1970 original, Princeton Paperbacks.

[30] E.D. Sontag, A Lyapunov-like characterization of asymptotic controllability, SIAM J. Control Optim. 21 (1983) $462-471$.

[31] E.D. Sontag, Stability and stabilization: discontinuities and the effect of disturbances, in: Nonlinear Analysis, Differential Equations and Control (Montreal, QC, 1998), Kluwer Academic, Dordrecht, 1999, pp. 307-367.

[32] E.D. Sontag, Clocks and insensitivity to small measurement errors, ESAIM Control Optim. Calc. Var. 4 (1999) $537-557$.

[33] H.J. Sussmann, Subanalytic sets and feedback control, J. Differential Equations 31 (1) (1979) 31-52.

\section{Further reading}

[26] L. Rifford, The stabilization problem: AGAS and SRS feedbacks, in: Optimal Control, Stabilization and Nonsmooth Analysis, Lecture Notes in Control and Information Sciences, vol. 301, Springer-Verlag, Heidelberg, 2004, pp. 173-184. 